\title{
Review Article \\ MRI in Glioma Immunotherapy: Evidence, Pitfalls, and Perspectives
}

\author{
Domenico Aquino, ${ }^{1}$ Andrea Gioppo, ${ }^{1,2}$ Gaetano Finocchiaro, ${ }^{3}$ Maria Grazia Bruzzone, ${ }^{1}$ and \\ Valeria Cuccarini ${ }^{1}$ \\ ${ }^{1}$ Neuroradiology Unit, Fondazione IRCCS Istituto Neurologico "Carlo Besta", Milan, Italy \\ ${ }^{2}$ Postgraduate School in Radiodiagnostics, Università degli Studi di Milano, Milan, Italy \\ ${ }^{3}$ Molecular Neuro-Oncology Unit, Fondazione IRCCS Istituto Neurologico "Carlo Besta", Milan, Italy \\ Correspondence should be addressed to Maria Grazia Bruzzone; maria.bruzzone@istituto-besta.it
}

Received 1 December 2016; Revised 6 February 2017; Accepted 2 March 2017; Published 20 April 2017

Academic Editor: Cristina Maccalli

Copyright (C) 2017 Domenico Aquino et al. This is an open access article distributed under the Creative Commons Attribution License, which permits unrestricted use, distribution, and reproduction in any medium, provided the original work is properly cited.

\begin{abstract}
Pseudophenomena, that is, imaging alterations due to therapy rather than tumor evolution, have an important impact on the management of glioma patients and the results of clinical trials. RANO (response assessment in neurooncology) criteria, including conventional MRI (cMRI), addressed the issues of pseudoprogression after radiotherapy and concomitant chemotherapy and pseudoresponse during antiangiogenic therapy of glioblastomas (GBM) and other gliomas. The development of cancer immunotherapy forced the identification of further relevant response criteria, summarized by the iRANO working group in 2015. In spite of this, the unequivocal definition of glioma progression by cMRI remains difficult particularly in the setting of immunotherapy approaches provided by checkpoint inhibitors and dendritic cells. Advanced MRI (aMRI) may in principle address this unmet clinical need. Here, we discuss the potential contribution of different aMRI techniques and their indications and pitfalls in relation to biological and imaging features of glioma and immune system interactions.
\end{abstract}

\section{Introduction}

Glioblastoma multiforme (GBM) is the most common primary brain tumor in adults [1] and carries a grim prognosis.

Infiltrative nature of diffuse gliomas makes it difficult to eliminate microscopic disease despite macroscopic gross total resection. Recurrence of GBM is inevitable, and the median overall survival (OS) time of GBM patients receiving the standard treatment, which consists of maximal safe resection followed by radiation and adjuvant temozolomide, is about $14-16$ months $[2,3]$. At recurrence, no standard approach has been established (further surgery, reirradiation, chemotherapy, and antiangiogenic therapy) and despite advances in treatment for GBM, the survival of patients has not significantly improved over the past two decades.

The central nervous system (CNS) has been traditionally considered an immune-privileged system; however, it has been proved that immune cells can cross the blood-brain barrier (BBB) to gain access to the brain parenchyma and can leave the CNS to reach the cervical lymph nodes. Considering that the immune system has access to the brain and that GBM expresses multiple tumor antigens that can be targeted by immunotherapeutic approaches, the development of immunotherapy has gained considerable interest over the last decade [4].

Converging data indicate that cancer epitopes can be recognized by the immune system and therefore an immune reaction can be mounted to erase or block tumor growth. Resistant tumor clones, grown under immune pressure, create an immune suppressive environment that leads to the formation of relevant tumor. These general observations also apply to brain tumors. Cancer immunotherapy strategies are aimed at reverting such immune suppression [5].

Novel immunotherapeutic strategies being investigated to treat glioblastoma can be broadly divided into three major classes: active immunotherapy, adoptive immunotherapy, 
and immunomodulatory strategies [6]. They include vaccination therapy targeted against specific tumor antigens or whole tumor lysate, adoptive cellular therapy with cytotoxic $\mathrm{T}$ lymphocytes, chimeric antigen receptors and bispecific T-cell engaging antibodies to bypass major histocompatibility complex restriction, aptamer therapy allowing a more efficient target delivery, and checkpoint blockade to release the tumor-mediated inhibition of the immune system. Within active immunotherapy, to enhance the immunogenicity of GBM, two approaches are used nowadays: (a) peptide immunotherapy where the target is a cancerspecific antigen like EGFRvIII (epidermal growth factor receptor) and (b) dendritic cell immunotherapy where dendritic cells act as antigen-presenting cells and can be pulsed with autologous tumor lysate or peptides from cytomegalovirus that is present in GBM but not in normal brain [7]. Checkpoint inhibitors that have been used to treat advanced tumors with durable remission in some cases are now in clinical trials in GBM patients: they facilitate effective antitumoral immune response as they suppress coinhibitory pathways activated by neoplasms to suppress $\mathrm{T}$-cell responses against tumor cells [8].

Initial data show prolonged OS (23 to 38 months) in GBM patients treated by vaccines [9]. Upcoming clinical trials' results will clarify the efficacy of different cancer immunotherapy approaches, in particular using checkpoint inhibitors. Due to the heterogeneity of glioblastoma, multiple treatment strategies of immunotherapy, in addition with conventional therapy, will be more likely to succeed.

Efficacy of therapy is assessed by clinical examination and magnetic resonance imaging (MRI). Pseudoprogression, that is, imaging features suggesting tumor progression that is not confirmed subsequently, occurs in up to $30 \%$ of patients within three months after radiochemotherapy [10-12]. Thus, considering pseudoprogression as true tumor progression (and conversely) could lead to an inappropriate change in therapy and errors in assessing the efficacy of novel treatments [13]. Pseudoprogression during immunotherapy seems to occur more often, and its timeframe remains to be defined, potentially differing by the class of immunotherapy given.

To address these issues, the iRANO (immunotherapy response assessment in neurooncology) committee redefined the response assessment criteria for patients with neuro-oncological malignancies undergoing immunotherapy: the "limbo" window when radiologic worsening does not suggest immunotherapy suspension has been widened to six months, after which true progression, if detected, should be backdated [14].

Conventional MRI (cMRI) has limitations in differentiating tumor progression/recurrence and immunotherapy responses [15]. Advanced MRI (aMRI) may allow a deeper understanding of tumor structure and biology. Unlike contrast enhancement, increased perfusion may be independent of $\mathrm{BBB}$ integrity and defines tumor neoangiogenesis [16]. On diffusion-weighted imaging (DWI), the apparent diffusion coefficient (ADC) inversely correlates to tumor cellularity [17]. MR spectroscopy (MRS) provides information about metabolites within tumoral and perilesional tissue [18].
Amino acid PET (mainly with methionine and fluoroethyltyrosine) has been used to enlighten the greater metabolic activity of malignant tumoral tissue compared to radionecrosis and might also help in differentiating progression from treatment-related alterations during immunotherapy [19-22]. Such facilities are restricted to a limited number of specialized centers. A review on amino acid PET, however, is beyond the aim of this review.

Evidence that aMRI techniques can differentiate pseudoprogression and tumor recurrence has been reported in radiotherapy and chemotherapy, and promising data suggest they may differentiate at early-stage responder and nonresponder patients to immunotherapy. The purpose of this review is to summarize current research on MRI assessment for patients undergoing immunotherapy with a major focus on aMRI parameters.

\section{Magnetic Resonance Imaging (MRI)}

2.1. Conventional MRI (cMRI). Several criteria have been proposed in the last two decades to assess response to therapy in gliomas: the standard method is based on contrast-enhancing images in $\mathrm{T} 1$ and on hyperintensity in $\mathrm{T} 2$ or FLAIR (fluid-attenuated inversion recovery) sequences. Nevertheless, enhancement on T1 reflects nonspecific impairment of the $\mathrm{BBB}$, a reduction or lack of enhancement can be due to tumor shrinkage but also to antiangiogenic therapy, due to vascular normalization besides tumor infiltration (pseudoresponse). On the other hand, in pseudoprogression, an early, subacute reaction to treatment (e.g., radiotherapy) is associated with contrast enhancement, edema, and possible mass effect, and sometimes, associated clinical symptoms initially suggest tumor progression but subsequently resolve without any further treatment and can be associated to longer survival [23]. Furthermore, T2 and FLAIR hyperintensity can be associated to tumor infiltration, but also to edema, ischemia, gliosis, demyelination, inflammation, or postactinic alteration. In particular, inflammation might mimic radiological features of tumor progression with increased enhancement including new lesions and edema.

Pseudoprogression is generally not associated with clinical deterioration in radiochemotherapy [13] but can be linked to increased edema and clinical symptoms during immunotherapy. Since effective immune responses might need time to develop, early imaging might reflect true progressive disease and only later be followed by delayed response. Notably, previous experience in melanomas showed that while tumor regression is often low (about $10 \%$ ), many patients could have prolonged periods of disease stabilization [24].

Volume of enhancement lacks to differentiate between progressive disease and pseudoprogression. Moreover, the pattern of enhancement in pseudoprogression is not specific and can be nonhomogeneous, mimicking GBM, nodular, "cottony," and sometimes quite intense as in "flare" inflammatory phenomena also observed after local intracerebral/ intratumoral immunotherapies [25].

RANO (response assessment in neurooncology) criteria, including cMRI were published in 2010 to address the issues 
of pseudoprogression after radiochemotherapy and/or pseudoresponse during antiangiogenic therapy [26]. To overcome limitations of previous criteria, T2/FLAIR assessment of the lesions was included, especially in patients treated by antiangiogenic antibodies like bevacizumab; pseudoprogression after radiotherapy was considered if recurrence was present in the radiated field within 12 weeks after the completion of radiotherapy and required a repeated scan after 4 weeks to confirm or exclude progression; corticosteroid use and clinical status were also considered. Current RANO criteria are based on two-dimensional measurements on MRI. However, there is an ongoing debate on to whether volumetric measurements would be more accurate in defining tumor evolution over time, and the inclusion of such measurements as secondary study endpoints is encouraged [19].

Pseudoprogression can be more frequent after immunotherapy. The precise mechanism of pseudoprogression, occurring in up to $30 \%$ of patients with glioblastoma after radiochemotherapy, is poorly understood $[12,27]$. In some immunotherapy cases, histopathology showed infiltration of CD8+ lymphocytes, but not mitotically active tumor cells [28]. Effective immune response might need time to evolve, and early imaging might reflect true progressive disease; on the other hand, inflammatory response in areas of macroscopic or microscopic infiltrative tumor might mimic radiological features of tumor progression with increased enhancement and edema.

In 2009 , the increased interest in evaluating immunotherapies led to the development of immune-related response criteria (irRC) [24]: these guidelines considered that inflammatory responses may imply transient enlargement of the tumor or the appearance of new lesions thus complicating the assessment of tumor progression and recommended that since new lesions do not necessarily indicate progression, patients with enlarging lesions should repeat the scan 4 weeks later.

The iRANO committee, integrating guidance for progressive imaging findings from the irRC with RANO criteria, redefined the response assessment criteria for patients with brain tumors undergoing immunotherapy providing novel iRANO criteria [14]: in patients with early findings suggesting progression (i.e., $\geq 25 \%$ increase in the sum of biperpendicular diameters of enhancing tissue, development of new lesions, or substantial worsening in T2/FLAIR) within the first 6 months of immunotherapy regimen without substantial neurological decline, therapy should be continued and confirmation of radiographic progression by follow-up imaging should be sought 3 months after the initial radiographic evidence of progressive disease (Table 1, Figure 1).

\subsection{Delayed-Contrast MRI: TRAMs (Treatment Response} Assessment Maps). In recent years, Zach et al. proposed a new method to distinguish active tumor and treatmentinduced effects [29]. The method implies the acquisition of two high-resolution 3D T1-weighted sequences in the same MR session, 3-5 and 60-75 minutes after the injection of the contrast medium and in the subsequent subtraction of early from late sequences. The map obtained is then color-coded to differently represent areas in which contrast
TABLE 1: iRANO criteria (modified from [14]).

RANO criteria for high-grade gliomas

(i) Disappearance of all enhancing disease for $\geq 4$ weeks AND

(ii) No new lesions AND

Complete response (CR)

(iii) Stable/improved T2/FLAIR AND

(iv) No more than physiologic steroids AND

(v) Stable/improved clinically

(i) $\geq 50 \% \downarrow$ sum of biperpendicular diameters of enhancing disease for $\geq 4$ weeks AND

Partial response (PR)

(ii) No new lesions AND

(iii) Stable/improved T2/FLAIR AND

(iv) Stable/improved steroids AND

(v) Stable/improved clinically

(i) Does not qualify for CR, PR, and PD AND

Stable disease (SD)

(ii) No new lesions AND

(iii) Stable/improved T2/FLAIR AND

(iv) Stable/improved steroids AND

(v) Stable/improved clinically

(i) $\geq 25 \% \uparrow$ sum of biperpendicular diameters of enhancing disease OR

Progressive disease (PD)

(ii) New lesions OR

(iii) Significant worsened T2/FLAIR OR

(iv) Significant clinical decline

\begin{tabular}{lcc}
\hline iRANO criteria & \\
\hline $\begin{array}{c}\text { If } \leq 6 \text { months } \\
\text { after start of IT }\end{array}$ & $\begin{array}{c}\text { If }>6 \text { months } \\
\text { after start of IT }\end{array}$
\end{tabular}

Is a repeat scan required

to confirm radiographic

PD for patients without

Yes No

significant clinical decline?

\begin{tabular}{|c|c|c|}
\hline $\begin{array}{l}\text { Minimal time interval for } \\
\text { confirmation of progression } \\
\text { for patients without } \\
\text { significant clinical decline }\end{array}$ & $\geq 3$ months & Not applicable \\
\hline $\begin{array}{l}\text { Is further immunotherapy } \\
\text { (IT) treatment allowed } \\
\text { after initial radiographic } \\
\text { PD (if clinically stable) } \\
\text { pending progression } \\
\text { confirmation? }\end{array}$ & Yes & Not applicable \\
\hline $\begin{array}{l}\text { Does a new lesion } \\
\text { define PD? }\end{array}$ & No & Yes \\
\hline
\end{tabular}

accumulates during time (red-coded) and regions in which contrast is rapidly cleared from the tissue (blue-coded). Histological validation allowed to identify blue regions as the active tumor regions and red areas as treatmentinduced regions in which vessel lumen resulted disrupted and contrast tended to accumulate. The maps obtained are defined as treatment response assessment maps (TRAMs). Semiautomated calculation of the volume of each component can be performed and longitudinally monitored.

Different from other methodologies, TRAMs are not user-dependent, less acquisition-dependent (i.e., they only 


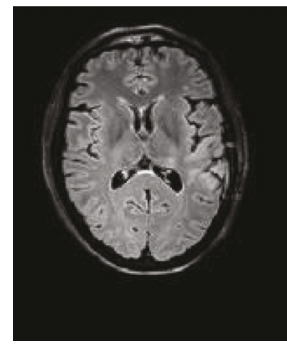

(a) Jun 2014

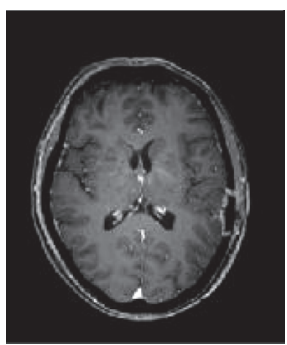

(f) Jun 2014

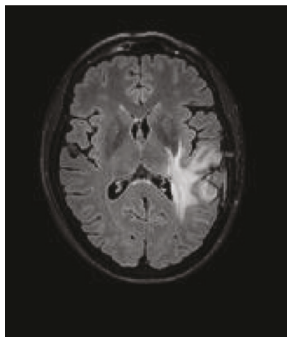

(b) Aug 2014

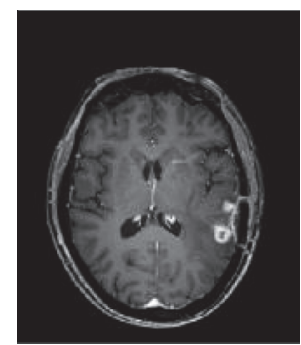

(g) Aug 2014

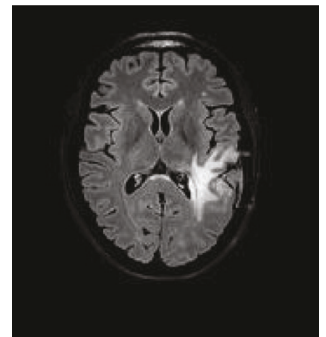

(c) Oct 2014

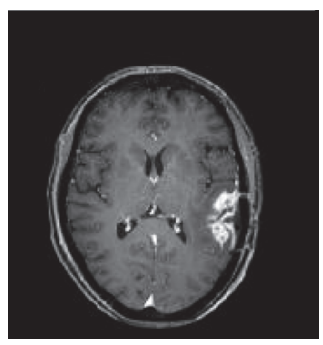

(h) Oct 2014

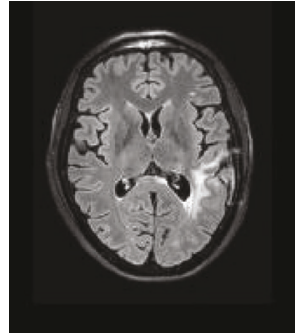

(d) Jan 2015

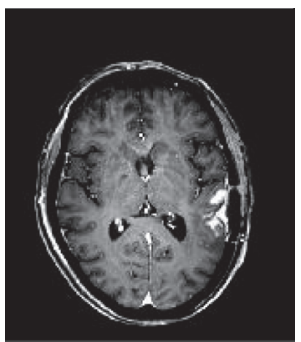

(i) Jan 2015

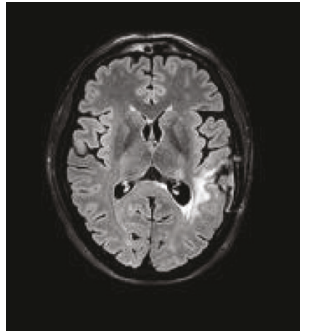

(e) Mar 2015

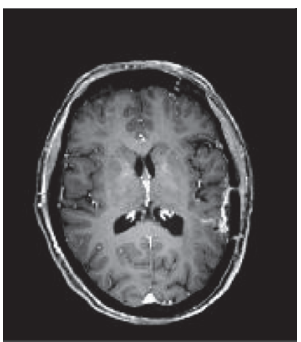

(j) Mar 2015

FIgURe 1: FLAIR (a-e) and contrast-enhanced T1-weighted images ( $f-j)$ : postsurgical $(a, f)$, increasing edema (b, c), enhancement (g, h) and subsequent reduction $(d, e, i)$ of both, and remission of the enhancing lesion $(j)$ in the course of immunotherapy with dendritic cell vaccine.

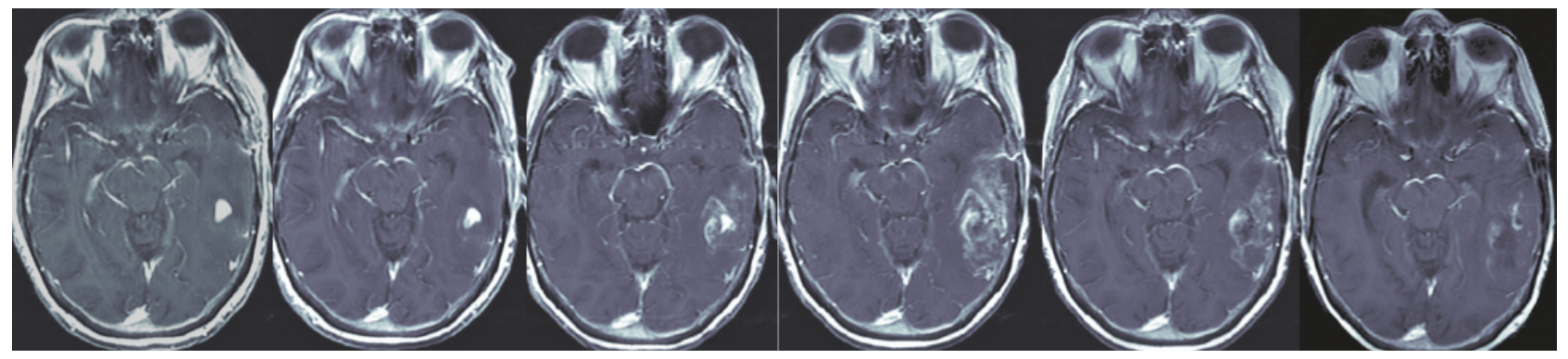

(a)

(b)

(c)

(d)

(e)

(f)

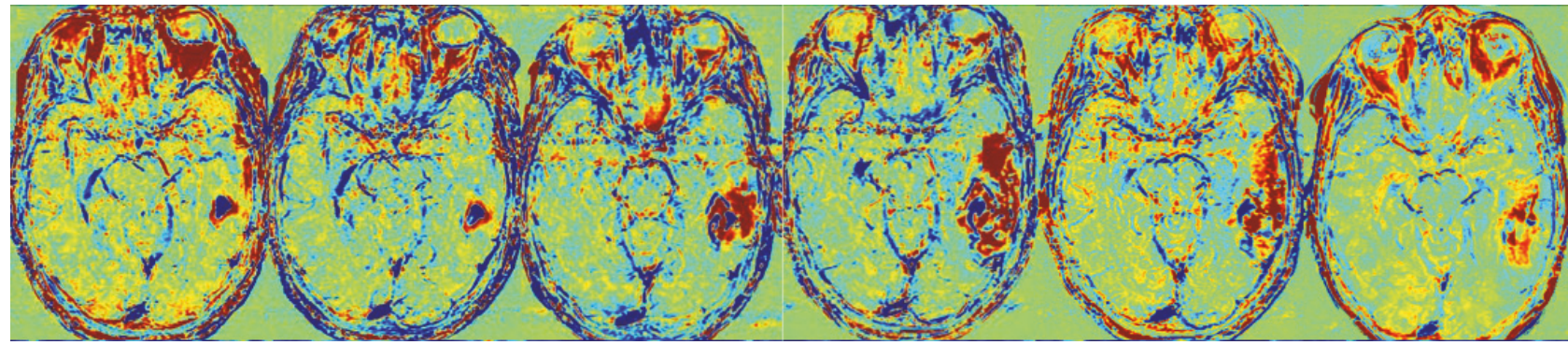

(g)

(h)

(i)

(j)

(k)

(1)

FIGURE 2: Contrast-enhanced T1-weighted images (a-f) and the calculated TRAM postchemoradiation (g-l) (images were acquired 0.7, 2.5, 4, 6,7 , and 8 months postchemoradiation). Temporary enlargement of enhancing lesion (c-d) is shown; as it can be seen, the red volume growth rate was prevalent above the blue volume $(\mathrm{i}-\mathrm{j})$, favoring pseudoprogression over progression. Pseudoprogression was later confirmed by the decrease in all volumes 7 and 8 months postchemoradiation (e-f, k-l). Modified from [110] with permission.

need good-quality 3D T1 sequences), and relatively simple to be acquired.

The inconveniences are that patient has to wait longer outside the scanner and that timing of postcontrast acquisitions is quite critical. The choice of the first time point is important because right after contrast injection, the gadolinium signal rises fast and the signal has to be high when the images are acquired in order to be sensitive to tumor regions (blue). On the other hand, this acquisition time point has to be early enough not to lose sensitivity to treatment effects (red). The closer to the maximal peak value, the larger is the difference between early and delayed signal 
and, consequently, sensitivity. For these reasons, 3-5 minutes should be used and, due to the fast changes in signal intensity, it is important to fix this time point for each patient follow-up. The choice of the second time point is mainly affected by the time the tumor takes to clear gadolinium from the tissue. Inter- and intratumor variability in clearance times exist, but after 1 hour, the signal changes slowly; therefore, the second time point can be flexible (to allow for a practical clinical application in a busy radiology department) between 1 and 1.45 hours postinjection.

2.2.1. Response Assessment. TRAMs have been used in radiochemotherapy and antiangiogenic therapy allowing discrimination between tumor and treatment-related effects (Figure 2) with sensitivity $100 \%$ and positive predictive value $92 \%$, demonstrating different TRAM patterns on pretreatment and early treatment stage in responder versus nonresponder patients $[29,30]$. In these studies, TRAMs showed higher accuracy than cerebral blood volume (CBV) in PWI (see Section 2.3 for details).

2.2.2. Immunotherapy. The rationale for applying TRAM analysis to immunotherapy lies on the differentiation between tumor and immune cells: preliminary data showed different components in enhancing lesions during immunotherapy with dendritic cells, with prevalence of "blue" regions in early progressive cases. However, longer follow-up in responder versus nonresponder patients is needed to understand if TRAMs can define immune-mediated pseudoprogression as they do in postradiotherapy follow-up.

2.3. Perfusion-Weighted Imaging (PWI). Three different MRI techniques have been developed to study brain microvascular hemodynamics. Two are based on the injection of gadolinium-based contrast medium, dynamic susceptibility contrast (DSC) and dynamic contrast enhanced (DCE), and the other, arterial spin labeling (ASL), uses blood as an internal contrast medium.

2.3.1. Dynamic Susceptibility Contrast (DSC)-MRI Perfusion. DSC [31] aims to study the hemodynamic characteristics of the brain microvascular network after the injection of a paramagnetic contrast medium and the contemporary dynamic acquisition of the brain volume. The estimation is obtained indirectly, starting from the signal intensity change caused by the passage of the contrast agent. The injection of a highly concentrated bolus is rapid (about $4-5 \mathrm{ml} / \mathrm{sec}$ ), in order to cause an appreciable signal decrease. The sequence is an echo-planar spin (SE)/gradient-echo (GRE), and DSC acquisition lasts 1-2 minutes.

After the acquisition, in the postprocessing step, a signaltime curve is extracted from every voxel of the brain volume. The curve is composed of a first baseline, prior to contrast arrival, a sharp peak corresponding to contrast bolus arrival and a final portion that represents contrast recirculation. Starting from the signal, the concentration of the contrast medium during time is mathematically obtained in every voxel. From every concentration-time curve obtained, four semiquantitative parameters can be derived: (a) CBV, defined as the ratio between the blood volume passed in a region and that entering that region [31], is an indirect measurement of the regional capillary bed density and an indicator of neoangiogenesis [32]; (b) cerebral blood flow $(\mathrm{CBF})$, representing microvascular blood flow rate in that region, is estimated by the concentration-time curve of the contrast entering the region to be examined called arterial input function (AIF), obtained by placing a region of interest (ROI) on one of the major arteries of the brain; (c) mean transit time (MTT), the time necessary to the contrast medium to pass through the area under examination; and (d) time to peak (TTP), the time necessary to reach the maximum contrast concentration.

CBV has found applications in the study of brain gliomas for tumor grading $[16,33,34]$, in the distinction between recurrence and radionecrosis [35] and in the prediction of clinical outcome and response $[36,37]$.

$\mathrm{BBB}$ disruption is a frequent condition in brain gliomas. In this condition, the contrast molecules can leak from vascular space and reach the parenchyma. This results in an unwanted T1-weighted effect leading to under- or overestimation of CBV that can be overcome by the injection of a prebolus of contrast to saturate T1.

The principal limitations of DSC are related to the sensitivity of the sequence to BBB disruption or susceptibility artifacts at natural interfaces (e.g., trabecular bone, paranasal sinuses, skull base, and sella), generally heavier at high field strengths.

\subsubsection{Dynamic Contrast Enhanced (DCE)-MRI Perfusion.}

This technique uses gadolinium to characterize the $\mathrm{BBB}$ and estimate its damage [38]. Different from DSC, the sequence acquired is T1-weighted (typically a 3D spoiled gradient-echo sequence) since, due to BBB disruption, contrast medium crosses the endothelial wall accumulating in the extravascular tissue causing T1 enhancement. DCE takes advantage of the signal intensity increase: dynamics is slower (acquisition time is $6-10 \mathrm{~min}$ ), due to the time for contrast medium to be washed in and out.

The first step is the conversion of enhancing signaltime curves into concentration-time curves. From the concentration-time curves, a first not specific index can be derived: the initial area under the curve (iAUC). Comparable to $\mathrm{CBV}$, this index could give indication about contrast leakage. Higher iAUC values generally correspond to more malignant conditions, where vessel permeability is high. This index however includes multiple information such as flow and permeability.

In order to have more detailed and specific information about BBB damage, the tissue in each voxel is represented by a multicompartment model composed of vascular, extravascular, and intracellular space. Using pharmacokinetic models [39], it is possible to estimate quantitative parameters to characterize the vascular damage: (1) $K_{\text {trans }}$, the most frequently used metric in tumor assessment representing the rate of transfer between plasma and extravascular tissue; (2) $K_{\mathrm{ep}}$, the transfer rate between extravascular tissue and plasma; (3) $V_{\mathrm{e}}$, the extracellular volume, inversely correlated to high cellularity and mitotic activity [40]; and (4) $V_{p}$, the fractional plasma volume [41]. 
With DCE, it is possible to extract quantitative values on $\mathrm{BBB}$ damage and intra- and extravascular volumes. Their estimation however is limited by water exchange phenomena, by the choice of the AIF, and by the robustness of the fitting procedure. Moreover, DCE uses a T1-weighted sequence that, different from DSC, is not affected by susceptibility artifacts. A $3 \mathrm{~T}$ scanner or higher is preferable.

2.3.3. Arterial Spin Labeling (ASL). This technique does not use any external contrast medium: the contrast is the blood entering the brain that, magnetically labeled, can be used to estimate the CBF [42]. The first applications of ASL used a long radiofrequency (RF) pulse simultaneously with a selection gradient to adiabatically invert the spins of the feeding arteries (continuous ASL, CASL). Recently, rather than a long RF pulse, a train of short RF pulses combined with a strong gradient has been used (pulsed CASL, pCASL) [43].

The main limitations are related to the intrinsically poor signal-to-noise ratio, due to the low fraction of free-water spins and to their relaxation. For this reason, the sequence generally used is echo-planar, allowing the acquisition and the averaging of multiple volumes.

2.3.4. Response Assessment. DSC has been used to evaluate early responses [44-46] to antiangiogenic therapy [47] and chemoradiation [48]. Parametric response maps (PRMs) of CBV [49] have been recently compared to a classical ROI-based approach in the identification of recurrent GBMs responsive to bevacizumab and irinotecan (antiangiogenics) [50]. In GBM, DSC has been used in combination with ASL and DTI (diffusion tensor imaging, see Section 2.4 for details) to discriminate between progression and pseudoprogression in radiochemotherapy [51, 52]. Pseudoprogression is typically characterized by reduction of $\mathrm{CBV}$, relative peak height, and increase of signal intensity recovery [53]. CBV $<1.75$ was predictive of pseudoprogression versus true progression [54].

DCE has been used for differential diagnosis [55] and tumor grading $[56,57]$. Prognostic value of $K_{\text {trans }}$ has been particularly investigated, showing a correlation between high baseline levels and poor PFS and OS [58]. $K_{\text {trans }}$ and CBV, interestingly, did not co-correlate with prognosis. In patients with recurrent GBM, mean values of $V_{\mathrm{p}}$ and $K_{\text {trans }}$ were significantly reduced in the pseudoprogression but not in tumor progression [59].

ASL has recently found application in the study of brain tumors [60-64]. A retrospective analysis [65] demonstrated higher sensibility of ASL-CBF than cMRI to identify tumor progression. ASL in combination with DSC was used to discriminate pseudoprogression and real progression in 117 newly diagnosed GBM treated with chemoradiation [51]. ASL produced eight (12.9\%) more accurate results than DSC alone. In progressive and stable GBMs studied by using DSC, DCE, and ASL, perfusional values (CBV, CBF, ASL$\mathrm{CBF}$, and $K_{\text {trans }}$ ) were higher in progressive lesions: the most accurate technique was DSC (CBV and CBF) [66].

2.3.5. Immunotherapy. DSC perfusion has been studied during immunotherapy confirming that elevation of $\mathrm{CBV}$ in a region with contrast enhancement supports the diagnosis of malignant tumor $[67,68]$.

In eight patients treated with dendritic cell immunotherapy [67], the maximum normalized lesional CBV resulted highest in progressing tumors, intermediate in preprogressing lesions, and lowest in stable cases. Although a clear correlation between CBV and pseudoprogression was not achieved due to the small number of cases, the authors support maximum CBV as a potential radiological marker to differentiate between immunotherapy-induced inflammatory response and GBM recurrence.

Interestingly, a mismatch between enhancing volumes and high CBV volumes has been described in 11/79 examinations in three of six immune-treated patients: the region with elevated CBV was never larger than the region with contrast enhancement. Histopathological evaluation in two cases showed malignant cells with numerous proliferating vessels with thrombosis or ruptures [68]. On the other hand, areas of enhancing tissue without hyperperfusion could be a sign of reactive, inflammatory changes due to immune-mediated BBB impairment: this hypothesis is supported by histopathology in immune-treated brain metastases of melanoma, showing reactive astrocytosis and scattered inflammatory and microglial cells surrounding isolated clusters of tumor cells [69].

DCE at 7T field strength has been used in immunotherapy studies in rat models of GBM: increased Ve was found in tumors responding to treatment due to tumor cell death and reduced proliferation, as indicated by the decreased growth index on histology. On the contrary, progressive lesions exhibited the greatest growth index and $\mathrm{Ve}$ was decreased with a tendency to reduce transvascular transport $\left(K_{\text {trans }}\right)[70]$.

As vessel permeability can be affected by inflammation, endothelial junctions become less tight, $K_{\text {trans }}$ higher. Clinical studies are ongoing to evaluate permeability in immunotherapy and to associate DCE parameters to pseudoprogression or true progression and to clinical outcome.

ASL has not yet been used in immunotherapy follow-up and would be an option in patients with renal failure and severe allergy to contrast agents, also limiting the potential risk of chronic contrast accumulation [71].

\subsection{Diffusion MRI}

2.4.1. Diffusion-Weighted Imaging (DWI). Brownian motion of water molecules inside the tissues brings water to diffuse in different brain compartments. This motion can be detected using a modified version of a spin-echo sequence that includes two strong gradients (diffusion gradients) [72]. The strength and geometrical characteristics of these gradients are summarized by a scalar value, the $b$ value. The aim of these gradients is to magnetically label the spins in some directions of the space so as to catch their motion and reconstruct tissue architecture. Being dynamic phenomena, the sequence used is an echo-planar sequence that lasts few minutes and needs the acquisition of two volumes at different $b$ values, generally 0 and $1000 \mathrm{~s} / \mathrm{mm}^{2}$. In DWI acquisition, one coefficient only is calculated, the 
ADC, a measure of the amount of water diffusivity inside the tissues. ADC is inversely proportional to the cellularity concentration [73]: vasogenic edema results in higher ADC values, with increased extracellular water content, whereas cell swelling produces low ADC values. ADC has been largely used to study brain gliomas [74] and also stroke and neurodegenerative disorders. Different from PWI, DWI measures are not affected by user-dependent parameters and ADC values are consistent if scanner parameters are controlled and remain the same. Increased ADC levels could indicate a first response to radiochemotherapy [75].

2.4.2. Diffusion Tensor Imaging (DTI). Different from DWI that only gives information about the amount of water displacement, diffusion tensor imaging allows to infer water-motion directionality [76]. With DTI, it is possible to characterize and reconstruct the main pathways of white matter fibers. The base sequence used is the same but is repeated in at least six noncollinear directions of the space [77]. For this reason, a DTI acquisition generally lasts about 10-12 min. By putting together the values obtained in each direction, it is possible to estimate the three preferential directions along which water moves in that tissue. From these directions, some quantitative parameters can be calculated: (a) fractional anisotropy (FA), an index describing directionality of the white matter fibers in the voxel; the higher the FA value (maximum corresponds to 1 , minimum to 0 ), the higher is the voxel directionality. If the value is low, the movement of water spins could be retained isotropic. (b) Mean diffusivity (MD), similar to ADC, represents the water diffusivity degree. (c) Axial diffusivity (AD), the value of the principal eigenvalue, that is, the degree of movement along the principal direction, strictly related to axonal damage. (d) Radial diffusivity (RD) represents water diffusivity in a direction transversal to the principal and related to the myelinization degree. These values are calculated point by point and maps are constructed.

The role of DTI metrics in tumoral characterization is debated. Some studies found that the tumoral core is characterized by low MD and high FA values [78], whereas peritumoral edema shows high MD and low FA values identifying FA as a marker of tumor infiltration [79]. In rat gliomas, low FA values were found in the center of the lesion, high FA values in the peritumoral rim, and high $\mathrm{MD}, \mathrm{RD}$, and $\mathrm{AD}$ in the perilesional edema [80]. Even if DTI has been used for tumor grading $[78,81,82]$, there is no uniform consensus about the role of its metrics in glioma characterization. This is probably due to limited standardization of the results and validation with histopathology [83].

Starting from the DTI metrics and from the directional information extracted in each voxel, it is possible to reconstruct three-dimensionally the pathways of white matter fibers. This technique, called tractography, is widely used in association with functional MRI before tumor resection [84].

2.4.3. Response Assessment. DWI has been widely performed to characterize brain gliomas and monitor radiochemotherapy or antiangiogenic therapy [85]. Functional diffusion maps (fDMs) [75] and histogram analysis need [86] to be highlighted. These techniques, which apply ADC maps to characterize the heterogeneity of therapy response, have been used to evaluate the efficacy of cytotoxic and antiangiogenic therapies $[85,87,88]$.

Progressive disease in high-grade gliomas, different from pseudoprogression, seems characterized by low ADC values due to hypercellularity $[76,77,79]$. ADC studies were often conducted in conjunction with other MRI modalities to improve the characterization of glioma heterogeneity [89-91].

DTI has increasingly been performed to study high-grade gliomas; histogram analysis and fDMs can provide early evidence of low-grade glioma modifications during chemotherapy with respect to cMRI (i.e., RANO criteria [14]), but only few studies have used it to discriminate progression and pseudoprogression [92-94]. DTI after radiochemotherapy shows elevated levels of FA in tumor progression compared to pseudoprogressing enhancing lesions; longitudinal DTI without segmentation was also proposed [94].

2.4.4. Immunotherapy. During immunotherapy, an inflammatory reaction is expected, carrying edema and reduced tumor cell density on one side but immune cell accumulation and hypercellularity on the other side as in other brain inflammatory diseases such as encephalitis or abscesses that exhibit lower ADC values than normal brain $[95,96]$.

In a pilot study on eight patients treated with dendritic cell immunotherapy, minimum ADC levels were lower in enhancing lesions at progression [67]. Furthermore, ADC levels within nonenhancing, FLAIR hyperintense regions were lower in preprogressive than in stable lesions. The parameter might be predictive of response: even if the small number of cases (three progressive diseases and five stable diseases, pseudoprogression not specified) did not allow the characterization of treatment-induced effects, the authors support ADC as a potential radiological marker to differentiate immunotherapy-induced inflammatory response and GBM progression.

Serial parametric response mapping of ADC in 21 children carrying pontine glioma treated by peptide-based vaccination following radiation therapy showed fractional decreased ADC in the four patients experiencing pseudoprogression [97].

Very recently, in a retrospective analysis of 10 recurrent GBM patients [98], intermediate ADC (IADC) volumes of interest (VOI) were able to discriminate the five patients with clinical benefit (i.e., without unequivocal clinical radiologic or histopathologic evidence of glioma progression for at least five months since trial onset) from the others. IADC VOI represented voxels within the FLAIR VOI having ADC in the range of highly cellular tumors $\left(0.7-1.1 \times 10^{-3} \mathrm{~mm}^{2} / \mathrm{s}\right)$. IADC VOI started to decrease in patients with clinical benefit three months on average after immunotherapy onset while in other patients, the value continued to increase.

DTI has been proposed in the follow-up of gliomas with treatments other than immunotherapy [90] and could be performed in longitudinal follow-up of selected patients. The main limit of this technique during immunotherapy may be the frequent presence of substantial edema due to inflammation. 
2.5. Magnetic Resonance Spectroscopy (MRS). MRS aims to study brain metabolism identifying and quantifying some relevant metabolites in a specific region. The water proton is most commonly used, as it is easy to implement in most of the scanners. Unlike MRI, which uses the twodimensional signals to derive images of the brain, MRS uses a monodimensional ${ }^{1} \mathrm{H}$ signal to estimate relative metabolite concentrations. Two are the principal MRS modalities: (a) chemical shift imaging (CSI) that gives a spatial distribution of the metabolites taking at the same time spectra deriving from multiple brain voxels (a grid) and (b) single voxel spectroscopy (SVS) that only acquires spectra from a little portion (VOI) of the brain. Both SVS and CSI do not cover the entire brain volume.

The most common metabolites investigated by MRS are (a) N-acetyl aspartate (NAA), a neuronal marker decreasing when neuronal integrity is affected; (b) choline (Cho), a marker of increased cellular turnover usually elevated in tumors and inflammatory processes; and (c) creatine $(\mathrm{Cr})$, which gives a measure of energy storage. In brain tumors, NAA results generally decreased and Cho increased, probably due to the membrane turnover. Other metabolites whose concentration generally changes in brain tumors are lactate, due to the anaerobic glycolysis; lipids, probably because of membrane disruption and necrosis; and myoinositol, associated to "crowding" of glial cells. Most recently, tumor characterization and therapeutic monitoring benefited from the possibility to study 2-hydroxyglutarate (2HG), an oncometabolite accumulating in tumors carrying isocitrate dehydrogenase (IDH) gene mutations [99-101]. Data indicate that IDH1 mutations are immunologically targetable [102]. In a translational model, vaccination with peptides encompassing the mutation in mice transgenic for human major histocompatibility complex (MHC) classes I and II caused MHC class-II-restricted antitumor immune responses based on CD4+ T-cells [102, 103].

Absolute quantitative MRS gives the concentration of the metabolites in a given voxel. Ratios of metabolite concentrations and metabolic maps (i.e., colorimetric maps reporting the single metabolite or ratio values in every voxel of the CSI grid), can be obtained.

2.5.1. Response Assessment. MRS has been used for glioma diagnosis, grading, and response monitoring [104, 105]. It may discriminate recurrent gliomas and radiation necrosis, but the most accurate parameters result in normalized Cho/NAA and Cho/Cr ratios with $88 \%$ and $83 \%$ sensitivity and $86 \%$ and $83 \%$ specificity, respectively $[66,106]$. Other studies [107], however, showed low levels of NAA and high levels of Cho in both tumor progression and pseudoprogression [90, 91, 107], in particular at early phases after radiation [108].

2.5.2. Immunotherapy. GBM is usually characterized by high concentration of Cho, decreased $\mathrm{Cr}$ and NAA, and presence of lipids in necrotic areas. Because of the inter- and intraindividual heterogeneity of high-grade gliomas, metabolite concentrations can vary considerably. MRS can detect the presence of high Cho levels (and Cho/Cr or Cho/NAA ratios) within enhancing and perifocal tissue thus enlightening the presence of glioma: specificity is high but mixed scenarios with coexistence of glioma and treatment alterations are frequent (Figure 3). Spectroscopic maps from multivoxel acquisitions are useful to monitor Cho distribution and concentration in the altered field of interest in longitudinal follow-up.

MRS findings were reported in two GBM patients after multimodal treatment with surgery, radiation, intralesional immunotherapy (IL-4 toxin), and chemotherapy: pseudoprogression was observed with extensive and increasing enhancement which nearly completely regressed after four to six months. In both patients, MRS did not show increased Cho within the enhancing areas [109].

A "harmonic" reduction of Cho, Cr, and NAA in the presence of lipids is usually associated with radionecrosis while lipids in the presence of elevated $\mathrm{Cho/Cr}$ ratio and low NAA suggest the presence of high-grade glioma. In immunotherapy, lipids have been described as substrate of NK T-cells [110] and the presence of a lipidic peak might be associated to a better immunotherapy response. Thus, MRS correlations with immunological findings can be interesting, given the relevance of $\mathrm{NK}$ responses in recurrent GBM receiving immunotherapy with dendritic cells [111].

2.6. Susceptibility-Weighted Imaging (SWI). Susceptibilityweighted imaging (SWI) is a tool for high-resolution imaging of the vasculature. The technique relies on the phase signal of a $\mathrm{T}^{*}$ sequence to amplify contrast between veins and brain tissue based on their susceptibility differences. The sequence is a fully flow compensated, long echo, 3D gradient-recalled-echo (GRE) pulse sequence, lasting about 5-7 minutes. Magnitude and phase volumes derived by sequence acquisition are combined together to produce a new image particularly sensitive to iron, calcium, ferritin, and venous blood [112]. Postcontrast SWI modification in relation to precontrast SWI indicates that signal alterations in tumors are not the result of calcification or subacute hemorrhage. Moreover, SWI has enlightened heterogeneity in enhancing lesions. The main advantages of SWI are high reproducibility and the gain of information on macro- and microvasculature without contrast medium.

SWI has a role in the evaluation of the vascular organization of brain gliomas and of neoangiogenesis that rapidly produces small, tortuous, and immature vessels leading to microbleedings and surrounding edema and also in the identification of tumor calcifications.

2.6.1. Response Assessment. SWI has been used for tumor grading [113-116] and differential diagnosis [117], analyzing the presence of the so-called "intratumoral susceptibility signals" (ITSS), defined as "low signal intensity and fine linear or dot-like structures, with or without conglomeration, seen within the tumor" [118] often present in GBM and absent in lymphomas [117]. Because hypointense signal on SWI images has been shown to reflect both vascularity and vascular integrity, this technique has a potential as a predictive marker and in assessing treatment effects and response to antiangiogenic treatments or radiotherapy [119-121]. 


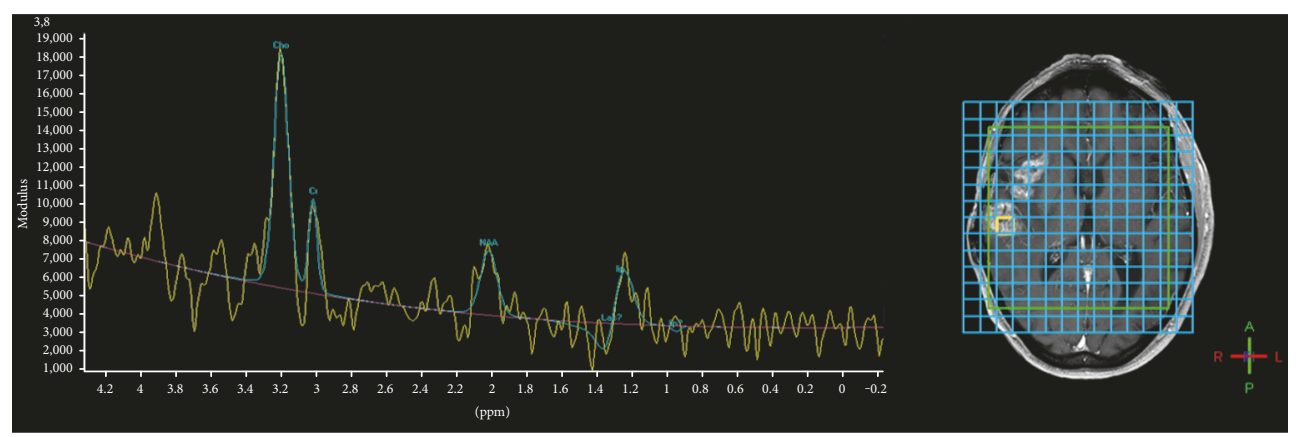

(a)

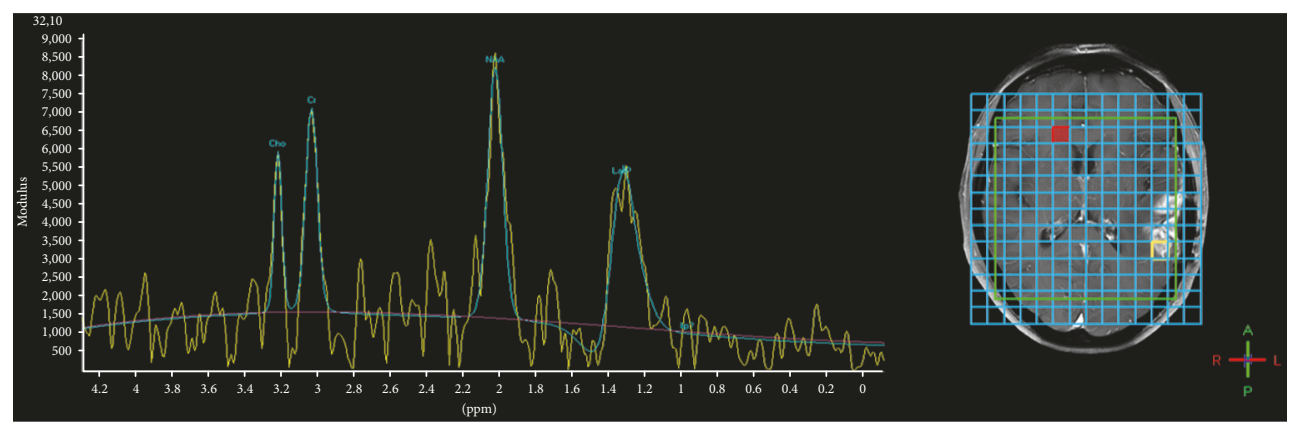

(b)

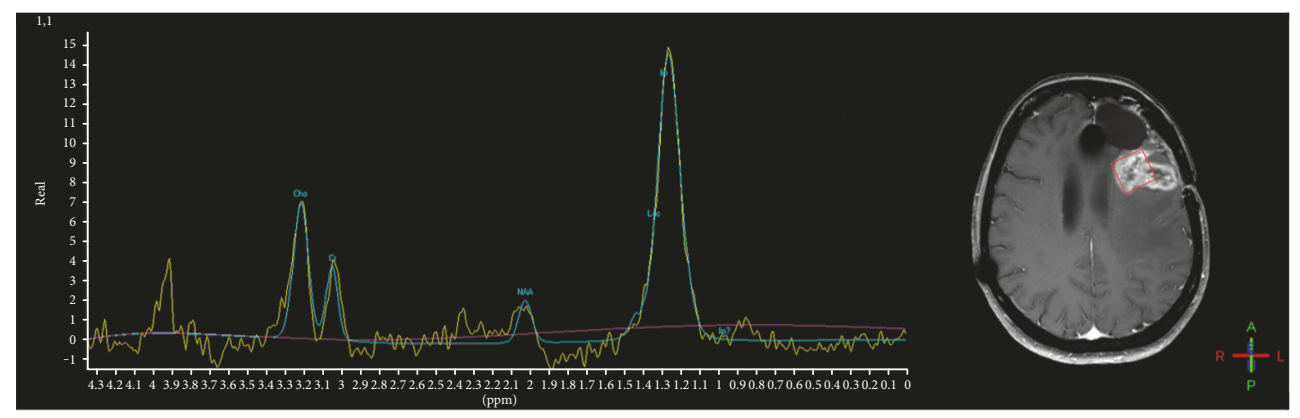

(c)

FIGURE 3: MRS during immunotherapy, after surgery, and radiotherapy plus temozolomide. Left, spectra; right, voxel positioned within enhancing lesions. (a) High Cho and low NAA with minimum lipids in recurrent glioma. (b) Preserved Cho and NAA levels with evident though not prevalent lipid peak in pseudoprogression (the same case is shown in Figure 1-time point Oct 2014). (c) Prominent peak of lipids and lower peaks of Cho, Cr, and NAA but with high $\mathrm{Cho/Cr}$ and Cho/NAA ratios in mixed scenario with glioma recurrence and radionecrosis.

Additionally, on SWI sequences, significant higher concentrations of gadolinium accumulate at the border of the tumoral invasion zone.

2.6.2. Immunotherapy. SWI data in immunotherapy have not yet been reported.

This technique, with contrast medium, might have a potential in immunotherapy response assessment as SWI features are a surrogate of vascularity and are more pronounced on lesional borders, where enhancement is frequently prominent in immune-treated tumors or inflammatory diseases. SWI might be considered to differentiate enhancing GBM from areas rich in immune cells, given that ITSS can be found in high-grade gliomas and the development of new ITTS suggests recurrent or progressive disease, features that are absent in lymphomas (i.e., hypercellular and lymphocytic tumors) [117] and not described in inflammatory conditions. Moreover, edema does not significantly interfere with SWI images.

\section{Concluding Remarks and Perspectives}

In spite of the improvement determined by RANO criteria first and iRANO subsequently, the imaging definition of the actual dynamics of glioma and immune cell interactions and their impact on patient survival during checkpoint or DC immunotherapy remains unsatisfactory.

Mixed scenarios with coexistence of glioma and treatment alterations are often the rule; moreover, with regard to the diagnostic specificity of contrast enhancement and of aMRI features, the situation after multimodal treatment could become confusing. It seems plausible that aMRI may 


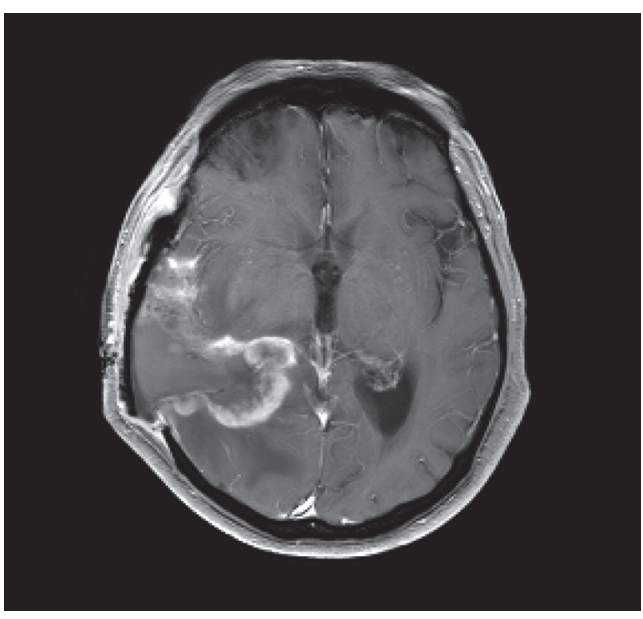

(a)

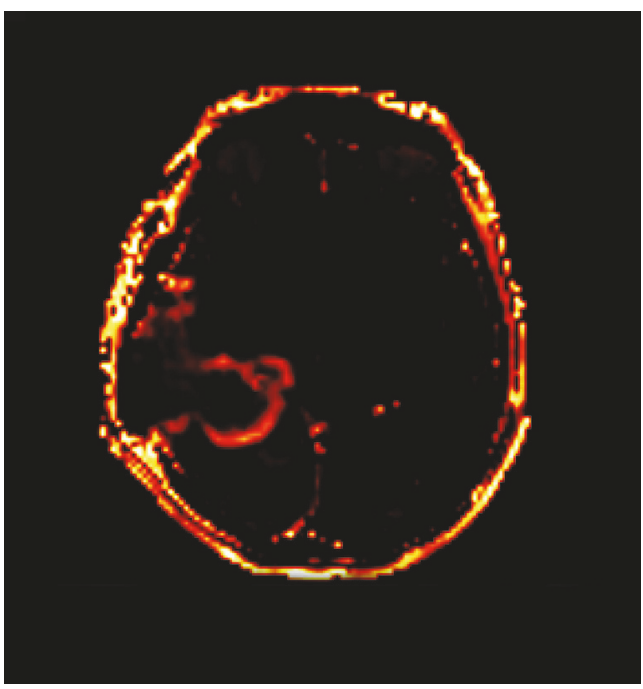

(c)

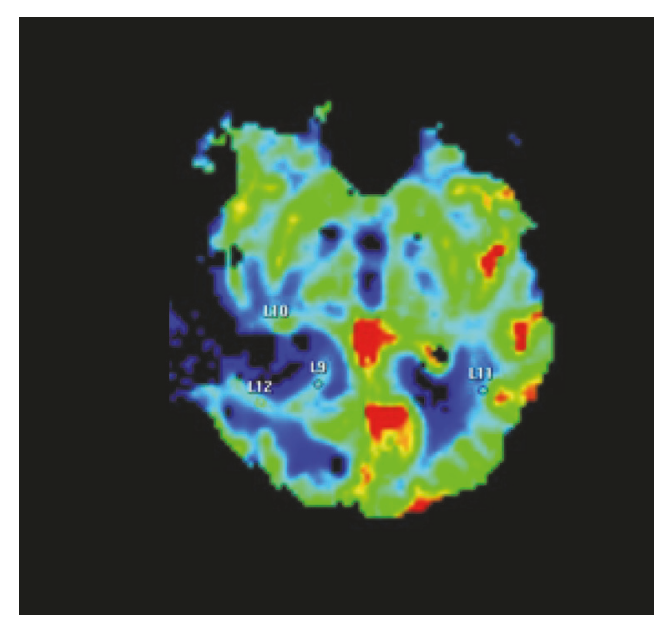

(b)

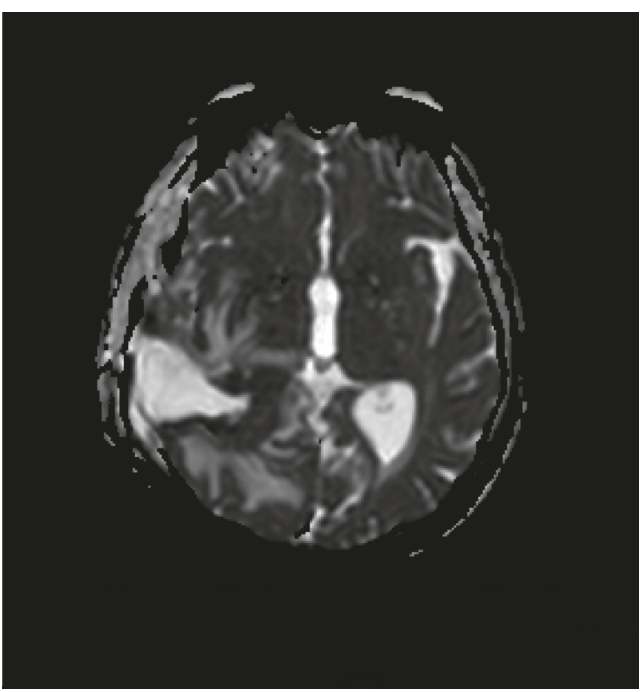

(d)

FIGURE 4: Enhancing lesion (a) during immunotherapy with dendritic cell vaccine. Mismatch between T1-enhancing volume and CBV (b), the last being just slightly elevated; permeability $\left(K_{\text {trans }}\right)$ is increased $(c)$. ADC is low $(d)$, suggesting hypercellularity.

provide deeper insights than cMRI in the recognition of pseudo or real glioma progression. On PWI, contrastenhancing areas secondary to immunotherapy inflammation should be less perfused than progressive/recurrent tumor, but also, vessel disruption and thrombosis due to high malignancy may inversely affect the perfusional pattern. Nevertheless, inflammation increases vessel permeability with effects on perfusional parameters. Likewise, low ADC can be associated to both tumoral and immune hypercellularity, and specific analysis has to be performed to discriminate. MRS is useful to obtain metabolic information within the enhanced areas, by determining high choline concentration and therefore identifying glioma within treatment alterations (Figure 3). Particular interest in pseudoprogression is focused on the mismatch between CBV and enhancing volumes, permeability parameters, ADC analysis (Figure 4), and presence of lipids as possible substrate of NK cells.

In clinical practice, a combination of different techniques may be necessary to differentiate between pseudoprogression and tumor progression. Cut-offs in a single-shot examination cannot distinguish between progression and pseudoprogression, and the evaluation of longitudinal modifications of parameters in terms of intensity and pattern is recommended. MRI data need to be analyzed taking into account that gliomas are generally composed of different structural and functional regions and that multimodal treatments increase brain tissue heterogeneity. Two are the main approaches used: (a) the histogram approach $[51,59,65,89,93,122-125]$ in which the evolution of the tumor during therapy is completely and quantitatively characterized estimating the statistical parameters (number of peaks, kurtosis, skewness, and other statistical moments) of the distribution of the values inside the lesion and (b) the voxel-based approach $[49,50,75,126]$, which aims to detect predictive markers of therapeutic efficacy estimating voxel by voxel the difference between the parametric maps (mainly $\mathrm{ADC}, \mathrm{CBV}$, and $K_{\text {trans }}$ ) of two different temporal points [75] [49]. An excellent example is that recently provided by Qin et al. in 2017 [98] based on an intermediate ADC (IADC) calculation in FLAIR VOI. 
In fact, the technique was able to discriminate patients with clinical benefit.

Imaging approaches like these, evolving in-depth analyses of MRI data that take into account whole-lesion heterogeneity and parametric modifications in the course of treatment such as parametric maps, TRAMs, and histogram analyses, deserve further investigation as they may provide the robust tools that are presently missing for the definition of PFS (i.e., progression-free survival) and clinical benefit in glioma immunotherapy.

\section{Abbreviations}

AD: $\quad$ Axial diffusivity

ADC: Apparent diffusion coefficient

aMRI: Advanced magnetic resonance imaging

ASL: Arterial spin labeling

BBB: Blood-brain barrier

CBF: Cerebral blood flow

CBV: Cerebral blood volume

Cho: Choline

cMRI: Conventional magnetic resonance imaging

CNS: Central nervous system

Cr: $\quad$ Creatine

DCE: Dynamic contrast enhanced

DSC: Dynamic susceptibility contrast

DTI: Diffusion tensor imaging

DWI: Diffusion-weighted imaging

FA: $\quad$ Fractional anisotropy

fDM: $\quad$ Functional diffusion maps

GBM: Glioblastoma multiforme

iAUC: Initial area under the curve

iRANO: Immunotherapy response assessment in neurooncology

ITSS: Intratumoral susceptibility signals

MD: $\quad$ Mean diffusivity

MRI: $\quad$ Magnetic resonance imaging

MRS: Magnetic resonance spectroscopy

NAA: $\quad \mathrm{N}$-acetyl aspartate

OS: Overall survival

PFS: Progression-free survival

PRM: Parametric response maps

RANO: Response assessment in neurooncology

$\mathrm{RD}: \quad$ Radial diffusivity

ROI: Region of interest

SWI: Susceptibility-weighted imaging

TRAMs: Treatment response assessment maps

Ve: $\quad$ Extracellular extravascular volume fraction

VOI: Volume of interest.

\section{Conflicts of Interest}

Gaetano Finocchiaro received an honorarium from BMS for the participation to the SNO 2016 advisory board.

\section{Authors' Contributions}

All authors have contributed to the work, concurred with the submission of the paper, and agreed with its content.

\section{References}

[1] J. Schuster, R. K. Lai, L. D. Recht et al., "A phase II, multicenter trial of rindopepimut (CDX-110) in newly diagnosed glioblastoma: the ACT III study," Neuro-Oncology, vol. 17, no. 6, pp. 854-861, 2015.

[2] R. Stupp, W. P. Mason, M. J. van den Bent et al., "Radiotherapy plus concomitant and adjuvant temozolomide for glioblastoma," The New England Journal of Medicine, vol. 352, no. 10, pp. 987-996, 2005.

[3] S. P. Weathers and M. R. Gilbert, "Current challenges in designing GBM trials for immunotherapy," Journal of Neuro-Oncology, vol. 123, no. 3, pp. 331-337, 2015.

[4] N. Kamran, A. Calinescu, M. Candolfi et al., "Recent advances and future of immunotherapy for glioblastoma," Expert Opinion on Biological Therapy, vol. 16, no. 10, pp. 1245-1264, 2016.

[5] B. D. Liebelt, G. Finocchiaro, and A. B. Heimberger, "Principles of immunotherapy," Handbook of Clinical Neurology, vol. 134, pp. 163-181, 2016.

[6] E. Ishikawa, K. Tsuboi, T. Yamamoto et al., "Clinical trial of autologous formalin-fixed tumor vaccine for glioblastoma multiforme patients," Cancer Science, vol. 98, no. 8, pp. 1226-1233, 2007.

[7] G. Finocchiaro and S. Pellegatta, "Novel mechanisms and approaches in immunotherapy for brain tumors," Discovery Medicine, vol. 20, no. 108, pp. 7-15, 2015, November 2016, http://www.ncbi.nlm.nih.gov/pubmed/26321082.

[8] M. Preusser, M. Lim, D. A. Hafler, D. A. Reardon, and J. H. Sampson, "Prospects of immune checkpoint modulators in the treatment of glioblastoma," Nature Reviews. Neurology, vol. 11, no. 9, pp. 504-514, 2015.

[9] L. W. Xu, K. K. H. Chow, M. Lim, and G. Li, "Current vaccine trials in glioblastoma: a review," Journal of Immunology Research, vol. 2014, Article ID 796856, p. 10, 2014.

[10] D. Brandsma, L. Stalpers, W. Taal, P. Sminia, and M. J. van den Bent, "Clinical features, mechanisms, and management of pseudoprogression in malignant gliomas," The Lancet Oncology, vol. 9, no. 5, pp. 453-461, 2008.

[11] A. Radbruch, J. Fladt, P. Kickingereder et al., "Pseudoprogression in patients with glioblastoma: clinical relevance despite low incidence," Neuro-Oncology, vol. 17, no. 1, pp. 151-159, 2015.

[12] A. A. Brandes, E. Franceschi, A. Tosoni et al., "MGMT promoter methylation status can predict the incidence and outcome of pseudoprogression after concomitant radiochemotherapy in newly diagnosed glioblastoma patients," Journal of Clinical Oncology, vol. 26, no. 13, pp. 2192-2197, 2008.

[13] S. Abdulla, J. Saada, G. Johnson, S. Jefferies, and T. Ajithkumar, "Tumour progression or pseudoprogression? A review of posttreatment radiological appearances of glioblastoma," Clinical Radiology, vol. 70, no. 11, pp. 1299-1312, 2015.

[14] H. Okada, M. Weller, R. Huang et al., "Immunotherapy response assessment in neuro-oncology: a report of the RANO working group," The Lancet Oncology, vol. 16, no. 15, pp. e534-e542, 2015.

[15] R. J. Young, A. Gupta, A. D. Shah et al., "Potential utility of conventional MRI signs in diagnosing pseudoprogression in glioblastoma," Neurology, vol. 76, no. 22, pp. 1918-1924, 2011. 
[16] M. Law, S. Yang, H. Wang et al., "Glioma grading: sensitivity, specificity, and predictive values of perfusion MR imaging and proton MR spectroscopic imaging compared with conventional MR imaging," AJNR. American Journal of Neuroradiology, vol. 24, no. 10, pp. 1989-1998, 2003, http://papers3://publication/uuid/C58DFDF1-444E-440BBD8E-55C3C32585DE.

[17] M.-A. Weber, F. L. Giesel, and B. Stieltjes, "MRI for identification of progression in brain tumors: from morphology to function," Expert Review of Neurotherapeutics, vol. 8, no. 10, pp. 1507-1525, 2008.

[18] J. H. Kim, K. H. Chang, D. G. Na et al., "3T 1H-MR spectroscopy in grading of cerebral gliomas: comparison of short and intermediate echo time sequences," American Journal of Neuroradiology, vol. 27, no. 7, pp. 1412-1418, 2006.

[19] E. Lopci, C. Franzese, M. Grimaldi et al., "Imaging biomarkers in primary brain tumours," European Journal of Nuclear Medicine and Molecular Imaging, vol. 42, no. 4, pp. 597-612, 2015.

[20] N. Galldiks, I. Law, W. B. Pope, J. Arbizu, and K.-J. Langen, "The use of amino acid PET and conventional MRI for monitoring of brain tumor therapy," NeuroImage Clinical, vol. 13, pp. 386-394, 2017.

[21] G. Pöpperl, C. Götz, W. Rachinger et al., "Serial O(2-[(18)F]fluoroethyl)-L: -tyrosine PET for monitoring the effects of intracavitary radioimmunotherapy in patients with malignant glioma," European Journal of Nuclear Medicine and Molecular Imaging, vol. 33, no. 7, pp. 792-800, 2006.

[22] Y. Chiba, M. Kinoshita, Y. Okita et al., "Use of (11)C-methionine PET parametric response map for monitoring WT1 immunotherapy response in recurrent malignant glioma," Journal of Neurosurgery, vol. 116, no. 4, pp. 835-842, 2012.

[23] T. J. Kruser, M. P. Mehta, and H. I. Robins, "Pseudoprogression after glioma therapy: a comprehensive review," Expert Review of Neurotherapeutics, vol. 13, no. 4, pp. 389-403, 2013.

[24] J. D. Wolchok, A. Hoos, S. O’Day et al., "Guidelines for the evaluation of immune therapy activity in solid tumors: immune-related response criteria," Clinical Cancer Research, vol. 15, no. 23, pp. 7412-7420, 2009.

[25] M. M. Smith, J. E. Thompson, M. Castillo et al., "MR of recurrent high-grade astrocytomas after intralesional immunotherapy," AJNR. American Journal of Neuroradiology, vol. 17, no. 6, pp. 1065-1071, 1996, November 2016, http://www.ncbi.nlm.nih.gov/pubmed/8791917.

[26] P. Y. Wen, D. R. Macdonald, D. A. Reardon et al., "Updated response assessment criteria for high-grade gliomas: response assessment in neuro-oncology working group," Journal of Clinical Oncology, vol. 28, no. 11, pp. 1963-1972, 2010.

[27] D.-S. Kong, S. T. Kim, E.-H. Kim et al., "Diagnostic dilemma of pseudoprogression in the treatment of newly diagnosed glioblastomas: the role of assessing relative cerebral blood flow volume and oxygen-6-methylguanine-DNA methyltransferase promoter methylation status," American Journal of Neuroradiology, vol. 32, no. 2, pp. 382-387, 2011.

[28] H. Okada, P. Kalinski, R. Ueda et al., "Induction of CD8+ $\mathrm{T}$-cell responses against novel glioma-associated antigen peptides and clinical activity by vaccinations with $\alpha$-type 1 polarized dendritic cells and polyinosinic-polycytidylic acid stabilized by lysine and carboxymethylcellulose in patients with recurrent malignant glioma," Journal of Clinical Oncology, vol. 29, no. 3, pp. 330-336, 2011.
[29] L. Zach, D. Guez, D. Last et al., "Delayed contrast extravasation MRI: a new paradigm in neuro-oncology," Neuro-Oncology, vol. 17, no. 3, pp. 457-465, 2015.

[30] D. Daniels, D. Guez, D. Last et al., "Early biomarkers from conventional and delayed-contrast MRI to predict the response to bevacizumab in recurrent high-grade gliomas," American Journal of Neuroradiology, vol. 37, no. 11, pp. 2003-2009, 2016.

[31] L. Ostergaard, A. G. Sorensen, K. K. Kwong, R. M. Weisskoff, C. Gyldensted, and B. R. Rosen, "High resolution measurement of cerebral blood flow using intravascular tracer bolus passages. Part II: experimental comparison and preliminary results," Magnetic Resonance in Medicine, vol. 36, no. 5, pp. 726-736, 1996, April 2016, http://www.ncbi.nlm.nih. gov/pubmed/8916023.

[32] J. Folkman, "Angiogenesis," Annual Review of Medicine, vol. 57, pp. 1-18, 2006.

[33] H. J. Aronen, I. E. Gazit, D. N. Louis et al., "Cerebral blood volume maps of gliomas: comparison with tumor grade and histologic findings," Radiology, vol. 191, no. 1, pp. 41-51, 1994.

[34] N. Morita, S. Wang, S. Chawla, H. Poptani, and E. R. Melhem, "Dynamic susceptibility contrast perfusion weighted imaging in grading of nonenhancing astrocytomas," Journal of Magnetic Resonance Imaging, vol. 32, no. 4, pp. 803-808, 2010.

[35] K. Mitsuya, Y. Nakasu, S. Horiguchi et al., "Perfusion weighted magnetic resonance imaging to distinguish the recurrence of metastatic brain tumors from radiation necrosis after stereotactic radiosurgery," Journal of NeuroOncology, vol. 99, no. 1, pp. 81-88, 2010.

[36] M. Law, S. Oh, J. S. Babb et al., "Low-grade gliomas: dynamic susceptibility-weighted contrast-enhanced perfusion MR imaging-prediction of patient clinical response," Radiology, vol. 238, no. 2, pp. 658-667, 2006.

[37] M. Law, R. J. Young, J. S. Babb et al., "Gliomas: predicting time to progression or survival with cerebral blood volume measurements," Radiology, vol. 247, no. 2, pp. 490-498, 2008.

[38] P. S. Tofts, "T1-weighted DCE imaging concepts: modelling, acquisition and analysis," MAGNETOM Flash, vol. 45, no. 3, pp. 30-39, 2010.

[39] P. S. Tofts and A. G. Kermode, "Measurement of the bloodbrain barrier permeability and leakage space using dynamic MR imaging. 1. Fundamental concepts," Magnetic Resonance in Medicine, vol. 17, no. 2, pp. 357-367, 1991.

[40] S. J. Mills, D. du Plessis, P. Pal et al., "Mitotic activity in glioblastoma correlates with estimated extravascular extracellular space derived from dynamic contrast-enhanced MR imaging," AJNR. American Journal of Neuroradiology, vol. 37, no. 5, pp. 811-817, 2016.

[41] A. K. Heye, R. D. Culling, M. C. del Valdés Hernández, M. J. Thrippleton, and J. M. Wardlaw, "Assessment of blood-brain barrier disruption using dynamic contrastenhanced MRI. A systematic review," NeuroImage Clinical, vol. 6, pp. 262-274, 2014.

[42] D. S. Williams, J. A. Detre, J. S. Leigh, and A. P. Koretsky, "Magnetic resonance imaging of perfusion using spin inversion of arterial water," Proceedings of the National Academy of Sciences of the United States of America, vol. 89, no. 1, pp. 212-216, 1992, May 2016, http://www.pubmedcentral. nih.gov/articlerender.fcgi?artid=48206\&tool=pmcentrez\& rendertype $=$ abstract. 
[43] W. Dai, D. Garcia, C. De Bazelaire, and D. C. Alsop, "Continuous flow-driven inversion for arterial spin labeling using pulsed radio frequency and gradient fields," Magnetic Resonance in Medicine, vol. 60, no. 6, pp. 1488-1497, 2008.

[44] T. T. Batchelor, E. R. Gerstner, K. E. Emblem et al., "Improved tumor oxygenation and survival in glioblastoma patients who show increased blood perfusion after cediranib and chemoradiation," Proceedings of the National Academy of Sciences of the United States of America, vol. 110, no. 47, pp. 19059-19064, 2013.

[45] R. F. Barajas, J. S. Chang, M. R. Segal et al., "Differentiation of recurrent glioblastoma multiforme from radiation necrosis after external beam radiation therapy with dynamic susceptibility-weighted contrast-enhanced perfusion MR imaging," Radiology, vol. 253, no. 2, pp. 486-496, 2009.

[46] L. S. Hu, L. C. Baxter, K. A. Smith et al., "Relative cerebral blood volume values to differentiate high-grade glioma recurrence from posttreatment radiation effect: direct correlation between image-guided tissue histopathology and localized dynamic susceptibility-weighted contrast-enhanced perfusion MR imaging measurements," American Journal of Neuroradiology, vol. 30, no. 3, pp. 552-558, 2009.

[47] E. R. Gerstner, K. E. Emblem, and G. A. Sorensen, "Vascular magnetic resonance imaging in brain tumors during antiangiogenic therapy - are we there yet?" The Cancer Journal, vol. 21, no. 4, pp. 337-342, 2015.

[48] R. Mangla, G. Singh, D. Ziegelitz et al., "Changes in relative cerebral blood volume 1 month after radiationtemozolomide therapy can help predict overall survival in patients with glioblastoma," Radiology, vol. 256, no. 2, pp. 575-584, 2010.

[49] C. J. Galbán, T. L. Chenevert, C. R. Meyer et al., "The parametric response map is an imaging biomarker for early cancer treatment outcome," Nature Medicine, vol. 15, no. 5, pp. 572-576, 2009.

[50] D. Aquino, A. L. Di Stefano, A. Scotti et al., "Parametric response maps of perfusion MRI may identify recurrent glioblastomas responsive to bevacizumab and irinotecan," PloS One, vol. 9, no. 3, article e90535, 2014.

[51] Y. J. Choi, H. S. Kim, G.-H. Jahng, S. J. Kim, and D. C. Suh, "Pseudoprogression in patients with glioblastoma: added value of arterial spin labeling to dynamic susceptibility contrast perfusion MR imaging," Acta Radiologica, vol. 54, no. 4, pp. 448-454, 2013.

[52] S. Wang, M. Martinez-Lage, Y. Sakai et al., "Differentiating tumor progression from pseudoprogression in patients with glioblastomas using diffusion tensor imaging and dynamic susceptibility contrast MRI," American Journal of Neuroradiology, vol. 37, no. 1, pp. 28-36, 2016.

[53] R. J. Young, A. Gupta, A. D. Shah et al., "MRI perfusion in determining pseudoprogression in patients with glioblastoma," Clinical Imaging, vol. 37, no. 1, pp. 41-49, 2013.

[54] S. Gahramanov, A. M. Raslan, L. L. Muldoon et al., "Potential for differentiation of pseudoprogression from true tumor progression with dynamic susceptibility-weighted contrastenhanced magnetic resonance imaging using ferumoxytol vs. gadoteridol: a pilot study," International Journal of Radiation Oncology, Biology, Physics, vol. 79, no. 2, pp. 514-523, 2011.

[55] A. Xyda, U. Haberland, E. Klotz et al., "Diagnostic performance of whole brain volume perfusion CT in intra-axial brain tumors: preoperative classification accuracy and histopathologic correlation," European Journal of Radiology, vol. 81, no. 12, pp. 4105-4111, 2012.

[56] T. B. Nguyen, G. O. Cron, K. Perdrizet et al., "Comparison of the diagnostic accuracy of DSC- and dynamic contrastenhanced MRI in the preoperative grading of astrocytomas," AJNR. American Journal of Neuroradiology, vol. 36, no. 11, pp. 2017-2022, 2015.

[57] C. Santarosa, A. Castellano, G. M. Conte et al., "Dynamic contrast-enhanced and dynamic susceptibility contrast perfusion MR imaging for glioma grading: preliminary comparison of vessel compartment and permeability parameters using hotspot and histogram analysis," European Journal of Radiology, vol. 85, no. 6, pp. 1147-1156, 2016.

[58] D. Bonekamp, K. Deike, B. Wiestler et al., "Association of overall survival in patients with newly diagnosed glioblastoma with contrast-enhanced perfusion MRI: comparison of intraindividually matched $\mathrm{T}_{1}$ - and $\mathrm{T}_{2}^{*}$-based bolus techniques," Journal of Magnetic Resonance Imaging, vol. 42, no. 1, pp. 87-96, 2015.

[59] A. A. Thomas, J. Arevalo-Perez, T. Kaley et al., "Dynamic contrast enhanced T1 MRI perfusion differentiates pseudoprogression from recurrent glioblastoma," Journal of NeuroOncology, vol. 125, no. 1, pp. 183-190, 2015.

[60] M.-A. Weber, A. Kroll, M. Günther et al., "Nichtinvasive Messung des relativen zerebralen Blutflusses mit der MR-Blutbolusmarkierungstechnik (arterial-spin-labeling): Physikalische Grundlagen und klinische Anwendungen," Radiologe, vol. 44, no. 2, pp. 164-174, 2004.

[61] J. Petr, I. Platzek, A. Seidlitz et al., "Early and late effects of radiochemotherapy on cerebral blood flow in glioblastoma patients measured with non-invasive perfusion MRI," Radiotherapy and Oncology, vol. 118, no. 1, pp. 24-28, 2016.

[62] D. Van Westen, E. T. Petersen, R. Wirestam et al., "Correlation between arterial blood volume obtained by arterial spin labelling and cerebral blood volume in intracranial tumours," Magnetic Resonance Materials in Physics, Biology and Medicine, vol. 24, no. 4, pp. 211-223, 2011.

[63] T. Abe, Y. Mizobuchi, W. Sako et al., "Clinical significance of discrepancy between arterial spin labeling images and contrast-enhanced images in the diagnosis of brain tumors," Magnetic Resonance in Medical Sciences, vol. 14, no. 4, pp. 313-319, 2015.

[64] C. M. White, W. B. Pope, T. Zaw et al., "Regional and voxelwise comparisons of blood flow measurements between dynamic susceptibility contrast magnetic resonance imaging (DSC-MRI) and arterial spin labeling (ASL) in brain tumors," Journal of Neuroimaging, vol. 24, no. 1, pp. 23-30, 2014.

[65] E. Nyberg, J. Honce, B. K. Kleinschmidt-DeMasters, B. Shukri, S. Kreidler, and L. Nagae, "Arterial spin labeling: pathologically proven superiority over conventional MRI for detection of high-grade glioma progression after treatment," The Neuroradiology Journal, vol. 29, no. 5, pp. 377-383, 2016.

[66] A. Seeger, C. Braun, M. Skardelly et al., "Comparison of three different MR perfusion techniques and MR spectroscopy for multiparametric assessment in distinguishing recurrent high-grade gliomas from stable disease," Academic Radiology, vol. 20, no. 12, pp. 1557-1565, 2013.

[67] M. Vrabec, S. Van Cauter, U. Himmelreich et al., "MR perfusion and diffusion imaging in the follow-up of recurrent glioblastoma treated with dendritic cell immunotherapy: a pilot study," Neuroradiology, vol. 53, no. 10, pp. 721-731, 2011. 
[68] L. Stenberg, E. Englund, R. Wirestam, P. Siesjo, L. G. Salford, and E.M.Larsson, "Dynamic susceptibility contrastenhanced perfusion magnetic resonance (MR) imaging combined with contrast-enhanced MR imaging in the follow-up of immunogene-treated glioblastoma multiforme," Acta Radiologica, vol. 47, no. 8, pp. 852-861, 2006.

[69] J. V. Cohen, A. K. Alomari, A. O. Vortmeyer et al., "Melanoma brain metastasis pseudoprogression after pembrolizumab treatment," Cancer Immunology Research, vol. 4, no. 3, pp. 179-182, 2016.

[70] C. Brekke Rygh, J. Wang, M. Thuen et al., "Dynamic contrast enhanced MRI detects early response to adoptive NK cellular immunotherapy targeting the NG2 proteoglycan in a rat model of glioblastoma," PloS One, vol. 9, no. 9, article e108414, 2014.

[71] R. J. McDonald, J. S. McDonald, D. F. Kallmes et al., "Intracranial gadolinium deposition after contrast-enhanced MR imaging," Radiology, vol. 275, no. 3, pp. 772-782, 2015.

[72] D. Le Bihan, R. Turner, P. Douek, and N. Patronas, "Diffusion MR imaging: clinical applications," AJR. American Journal of Roentgenology, vol. 159, no. 3, pp. 591-599, 1992.

[73] T. Sugahara, Y. Korogi, M. Kochi et al., "Usefulness of diffusion-weighted MRI with echo-planar technique in the evaluation of cellularity in gliomas," Journal of Magnetic Resonance Imaging, vol. 9, no. 1, pp. 53-60, 1999.

[74] F. W. Crawford and S. J. Nelson, "Evaluation of MR markers that predict survival in patients with newly diagnosed GBM prior to adjuvant therapy," Perfusion, vol. 91, no. 1, pp. 69-81, 2011.

[75] B. A. Moffat, T. L. Chenevert, T. S. Lawrence et al., "Functional diffusion map: a noninvasive MRI biomarker for early stratification of clinical brain tumor response," in Proceedings of the National Academy of Sciences of the United States of America, vol 102, no. 15, pp. 5524-5529, 2005.

[76] P. J. Basser, J. Mattiello, and D. LeBihan, "MR diffusion tensor spectroscopy and imaging," Biophysical Journal, vol. 66, no. 1, pp. 259-267, 1994.

[77] J. Hrabe, G. Kaur, and D. N. Guilfoyle, "Principles and limitations of NMR diffusion measurements," Journal of Medical Physics, vol. 32, no. 1, pp. 34-42, 2007.

[78] V. Z. Miloushev, D. S. Chow, and C. G. Filippi, "Metaanalysis of diffusion metrics for the prediction of tumor grade in gliomas," American Journal of Neuroradiology, vol. 36, no. 2, pp. 302-308, 2015.

[79] P. C. Sundgren, X. Fan, P. Weybright et al., "Differentiation of recurrent brain tumor versus radiation injury using diffusion tensor imaging in patients with new contrast-enhancing lesions," Magnetic Resonance Imaging, vol. 24, no. 9, pp. 1131-1142, 2006.

[80] S. Wang and J. Zhou, "Diffusion tensor magnetic resonance imaging of rat glioma models: a correlation study of MR imaging and histology," Journal of Computer Assisted Tomography, vol. 36, no. 6, pp. 739-744, 2012.

[81] W. Yuan, S. K. Holland, B. V. Jones, K. Crone, and F. T. Mangano, "Characterization of abnormal diffusion properties of supratentorial brain tumors: a preliminary diffusion tensor imaging study," Journal of Neurosurgery. Pediatrics, vol. 1, no. 4, pp. 263-269, 2008.

[82] A. Jakab, P. Molnár, M. Emri, and E. Berényi, “Glioma grade assessment by using histogram analysis of diffusion tensor imaging-derived maps," Neuroradiology, vol. 53, no. 7, pp. 483-491, 2011.
[83] A. Castellano and A. Falini, "Progress in neuro-imaging of brain tumors," Current Opinion in Oncology, vol. 28, no. 6, pp. 484-493, 2016.

[84] V. Baliyan, C. J. Das, R. Sharma, and A. K. Gupta, "Diffusion weighted imaging: technique and applications," World Journal of Radiology, vol. 8, no. 9, pp. 785-798, 2016.

[85] K. M. Schmainda, "Diffusion-weighted MRI as a biomarker for treatment response in glioma," CNS Oncology, vol. 1, no. 2, pp. 169-180, 2012.

[86] M. Nowosielski, W. Recheis, G. Goebel et al., "ADC histograms predict response to anti-angiogenic therapy in patients with recurrent high-grade glioma," Neuroradiology, vol. 53, no. 4, pp. 291-302, 2011.

[87] B. M. Ellingson, T. F. Cloughesy, A. Lai et al., "Graded functional diffusion map-defined characteristics of apparent diffusion coefficients predict overall survival in recurrent glioblastoma treated with bevacizumab," Neuro-Oncology, vol. 13, no. 10, pp. 1151-1161, 2011.

[88] R. Rahman, A. Hamdan, R. Zweifler et al., "Histogram analysis of apparent diffusion coefficient within enhancing and nonenhancing tumor volumes in recurrent glioblastoma patients treated with bevacizumab," Journal of NeuroOncology, vol. 119, no. 1, pp. 149-158, 2014.

[89] Y. S. Song, S. H. Choi, C. K. Park et al., “True progression versus pseudoprogression in the treatment of glioblastomas: a comparison study of normalized cerebral blood volume and apparent diffusion coefficient by histogram analysis," Korean Journal of Radiology, vol. 14, no. 4, pp. 662-672, 2013.

[90] M. Bulik, T. Kazda, P. Slampa, and R. Jancalek, "The diagnostic ability of follow-up imaging biomarkers after treatment of glioblastoma in the temozolomide era: implications from proton MR spectroscopy and apparent diffusion coefficient mapping," BioMed Research International, vol. 2015, Article ID 641023, p. 9, 2015.

[91] T. Kazda, M. Bulik, P. Pospisil et al., "Advanced MRI increases the diagnostic accuracy of recurrent glioblastoma: single institution thresholds and validation of MR spectroscopy and diffusion weighted MR imaging," NeuroImage Clinical, vol. 11, pp. 316-321, 2016.

[92] H. Kashimura, T. Inoue, T. Beppu, K. Ogasawara, and A. Ogawa, "Diffusion tensor imaging for differentiation of recurrent brain tumor and radiation necrosis after radiotherapy-three case reports," Clinical Neurology and Neurosurgery, vol. 109, no. 1, pp. 106-110, 2007.

[93] S. Wang, M. Martinez-Lage, Y. Sakai et al., "Differentiating tumor progression from pseudoprogression in patients with glioblastomas using diffusion tensor imaging and dynamic susceptibility contrast MRI," AJNR. American Journal of Neuroradiology, vol. 37, no. 1, pp. 28-36, 2016.

[94] X. Qian, H. Tan, J. Zhang, W. Zhao, M. D. Chan, and X. Zhou, "Stratification of pseudoprogression and true progression of glioblastoma multiform based on longitudinal diffusion tensor imaging without segmentation," Medical Physics, vol. 43, no. 11, pp. 5889-5902, 2016.

[95] V. Sawlani, "Diffusion-weighted imaging and apparent diffusion coefficient evaluation of herpes simplex encephalitis and Japanese encephalitis," Journal of the Neurological Sciences, vol. 287, no. 1-2, pp. 221-226, 2009.

[96] G. Luthra, A. Parihar, K. Nath et al., "Comparative evaluation of fungal, tubercular, and pyogenic brain abscesses with conventional and diffusion MR imaging and proton MR 
spectroscopy," American Journal of Neuroradiology, vol. 28, no. 7, pp. 1332-1338, 2007.

[97] R. Ceschin, B. F. Kurland, S. R. Abberbock et al., "Parametric response mapping of apparent diffusion coefficient as an imaging biomarker to distinguish pseudoprogression from TrueTumor progression in peptide-based vaccine therapy for pediatric diffuse intrinsic pontine glioma," American Journal of Neuroradiology, vol. 36, no. 11, pp. 2170-2176, 2015.

[98] L. Qin, X. Li, A. Stroiney et al., “Advanced MRI assessment to predict benefit of anti-programmed cell death 1 protein immunotherapy response in patients with recurrent glioblastoma," Neuroradiology, vol. 59, no. 2, pp. 1-11, 2017.

[99] O. C. Andronesi, F. Loebel, W. Bogner et al., "Treatment response assessment in IDH-mutant glioma patients by noninvasive 3D functional spectroscopic mapping of 2hydroxyglutarate," Clinical Cancer Research, vol. 22, no. 7, pp. 1632-1641, 2016.

[100] C. Choi, J. M. Raisanen, S. K. Ganji et al., "Prospective longitudinal analysis of 2-hydroxyglutarate magnetic resonance spectroscopy identifies broad clinical utility for the management of patients with IDH-mutant glioma," Journal of Clinical Oncology, vol. 34, no. 33, pp. 4030-4039, 2016.

[101] D. N. Louis, A. Perry, G. Reifenberger et al., "The 2016 World Health Organization classification of tumors of the central nervous system: a summary," Acta Neuropathologica, vol. 131, no. 6, pp. 803-820, 2016.

[102] G. Finocchiaro and S. Pellegatta, "Perspectives for immunotherapy in glioblastoma treatment," Current Opinion in Oncology, vol. 26, no. 6, pp. 608-614, 2014.

[103] A. Pajot, M.-L. Michel, N. Fazilleau et al., "A mouse model of human adaptive immune functions: HLA-A2.1-/HLA-DR1transgenic H-2 class I-/class II-knockout mice HLA class I and II transgenic mice/H-2 class I and II KO mice/CTL/ HTL/human immune response," European Journal of Immunology, vol. 34, no. 11, pp. 3060-3069, 2004.

[104] J. Kalpathy-Cramer, E. R. Gerstner, K. E. Emblem, O. C. Andronesi, and B. Rosen, "Advanced magnetic resonance imaging of the physical processes in human glioblastoma," Cancer Research, vol. 74, no. 17, pp. 4622-4637, 2014.

[105] S. J. Nelson, "Assessment of therapeutic response and treatment planning for brain tumors using metabolic and physiological MRI," NMR in Biomedicine, vol. 24, no. 6, pp. 734-749, 2011.

[106] H. Zhang, L. Ma, Q. Wang, X. Zheng, C. Wu, and B. Xu, "Role of magnetic resonance spectroscopy for the differentiation of recurrent glioma from radiation necrosis: a systematic review and meta-analysis," European Journal of Radiology, vol. 83, no. 12, pp. 2181-2189, 2014.

[107] L. C. Hygino da Cruz, I. Rodriguez, R. C. Domingues, E. L. Gasparetto, and A. G. Sorensen, "Pseudoprogression and pseudoresponse: imaging challenges in the assessment of posttreatment glioma," American Journal of Neuroradiology, vol. 32, no. 11, pp. 1978-1985, 2011.

[108] T. Kaminaga and K. Shirai, "Radiation-induced brain metabolic changes in the acute and early delayed phase detected with quantitative proton magnetic resonance spectroscopy," Journal of Computer Assisted Tomography, vol. 29, no. 3, pp. 293-297, 2005, January 2017, http://www.ncbi.nlm.nih. gov/pubmed/15891493.

[109] F. W. Floeth, H. J. Wittsack, V. Engelbrecht, and F. Weber, "Comparative follow-up of enhancement phenomena with
MRI and proton MR spectroscopic imaging after intralesional immunotherapy in glioblastoma - report of two exceptional cases," Zentralblatt für Neurochirurgie, vol. 63, no. 1, pp. 23-28, 2002.

[110] V. Lawson, "Turned on by danger: activation of CD1drestricted invariant natural killer T cells," Immunology, vol. 137, no. 1, pp. 20-27, 2012.

[111] S. Pellegatta, M. Eoli, S. Frigerio et al., "The natural killer cell response and tumor debulking are associated with prolonged survival in recurrent glioblastoma patients receiving dendritic cells loaded with autologous tumor lysates," Oncoimmunology, vol. 2, no. 3, article e23401, 2013.

[112] E. M. Haacke, S. Mittal, Z. Wu, J. Neelavalli, and Y.-C. N. Cheng, "Susceptibility-weighted imaging: technical aspects and clinical applications, part 1," AJNR. American Journal of Neuroradiology, vol. 30, no. 1, pp. 19-30, 2009.

[113] W. Mohammed, H. Xunning, S. Haibin, and M. Jingzhi, "Clinical applications of susceptibility-weighted imaging in detecting and grading intracranial gliomas: a review," Cancer Imaging, vol. 13, no. 2, pp. 186-195, 2013.

[114] C. Li, B. Ai, Y. Li, H. Qi, and L. Wu, "Susceptibility-weighted imaging in grading brain astrocytomas," European Journal of Radiology, vol. 75, no. 1, pp. e81-e85, 2010.

[115] X. Li, Y. Zhu, H. Kang et al., "Glioma grading by microvascular permeability parameters derived from dynamic contrast-enhanced MRI and intratumoral susceptibility signal on susceptibility weighted imaging," Cancer Imaging, vol. 15, no. 1, p. 4, 2015.

[116] Y. Ding, Z. Xing, B. Liu, X. Lin, and D. Cao, "Differentiation of primary central nervous system lymphoma from highgrade glioma and brain metastases using susceptibilityweighted imaging," Brain and Behavior: A Cognitive Neuroscience Perspective, vol. 4, no. 6, pp. 841-849, 2014.

[117] C. C. T. Hsu, T. W. Watkins, G. N. C. Kwan, and E. M. Haacke, "Susceptibility-weighted imaging of glioma: update on current imaging status and future directions," Journal of Neuroimaging, vol. 26, no. 4, pp. 383-390, 2016.

[118] M. J. Park, H. S. Kim, G. H. Jahng, C. W. Ryu, S. M. Park, and S. Y. Kim, "Semiquantitative assessment of intratumoral susceptibility signals using non-contrast-enhanced highfield high-resolution susceptibility-weighted imaging in patients with gliomas: comparison with MR perfusion imaging," American Journal of Neuroradiology, vol. 30, no. 7, pp. 1402-1408, 2009.

[119] W. Bian, C. P. Hess, S. M. Chang, S. J. Nelson, and J. M. Lupo, "Susceptibility-weighted MR imaging of radiation therapyinduced cerebral microbleeds in patients with glioma: a comparison between 3T and 7T," Neuroradiology, vol. 56, no. 2, pp. 91-96, 2014.

[120] J. M. Lupo, E. Essock-Burns, A. M. Molinaro et al., "Using susceptibility-weighted imaging to determine response to combined anti-angiogenic, cytotoxic, and radiation therapy in patients with glioblastoma multiforme," Neuro-Oncology, vol. 15, no. 4, pp. 480-489, 2013.

[121] S. Peters, R. Pahl, A. Claviez, and O. Jansen, "Detection of irreversible changes in susceptibility-weighted images after whole-brain irradiation of children," Neuroradiology, vol. 55, no. 7, pp. 853-859, 2013.

[122] H. J. Baek, H. S. Kim, N. Kim, Y. J. Choi, and Y. J. Kim, "(Histographic pattern) percent change of perfusion skewness and kurtosis: a potential imaging biomarker for early 
treatment response in patients with newly diagnosed glioblastomas," Radiology, vol. 264, no. 3, pp. 834-843, 2012.

[123] H. H. Chu, S. H. Choi, I. Ryoo et al., "Differentiation of true progression from pseudoprogression in glioblastoma treated with radiation therapy and concomitant temozolomide: comparison study of standard and high-b-value diffusion-weighted imaging," Radiology, vol. 269, no. 3, pp. 831-840, 2013.

[124] W. B. Pope, H. J. Kim, J. Huo et al., "Recurrent glioblastoma multiforme: ADC histogram analysis predicts response to bevacizumab treatment," Radiology, vol. 252, no. 1, pp. 182-189, 2009.

[125] M. Nowosielski, W. Recheis, G. Goebel et al., “ADC histograms predict response to anti-angiogenic therapy in patients with recurrent high-grade glioma," Neuroradiology, vol. 53, no. 4, pp. 291-302, 2011.

[126] C. Tsien, C. J. Galbán, T. L. Chenevert et al., "Parametric response map as an imaging biomarker to distinguish progression from pseudoprogression in high-grade glioma," Journal of Clinical Oncology, vol. 28, no. 13, pp. 2293-2299, 2010. 


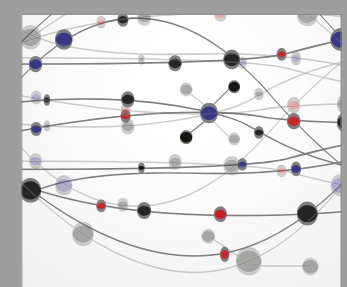

The Scientific World Journal
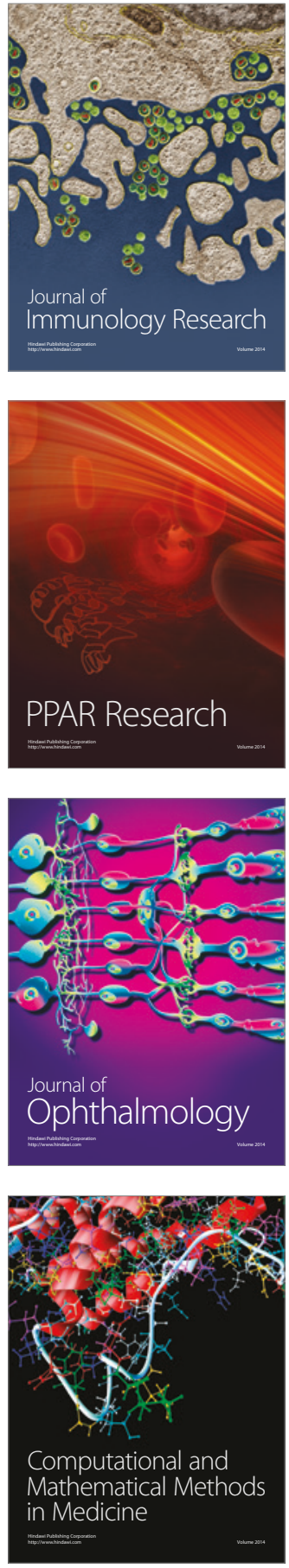

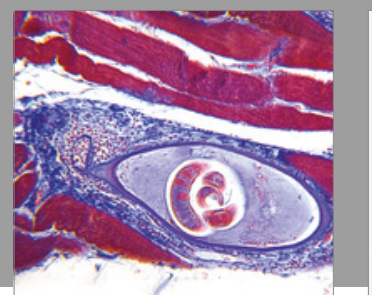

Gastroenterology Research and Practice
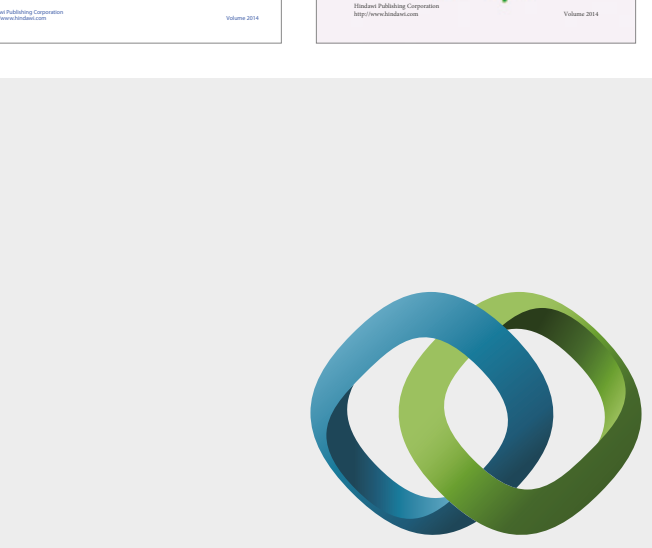

\section{Hindawi}

Submit your manuscripts at

https://www.hindawi.com
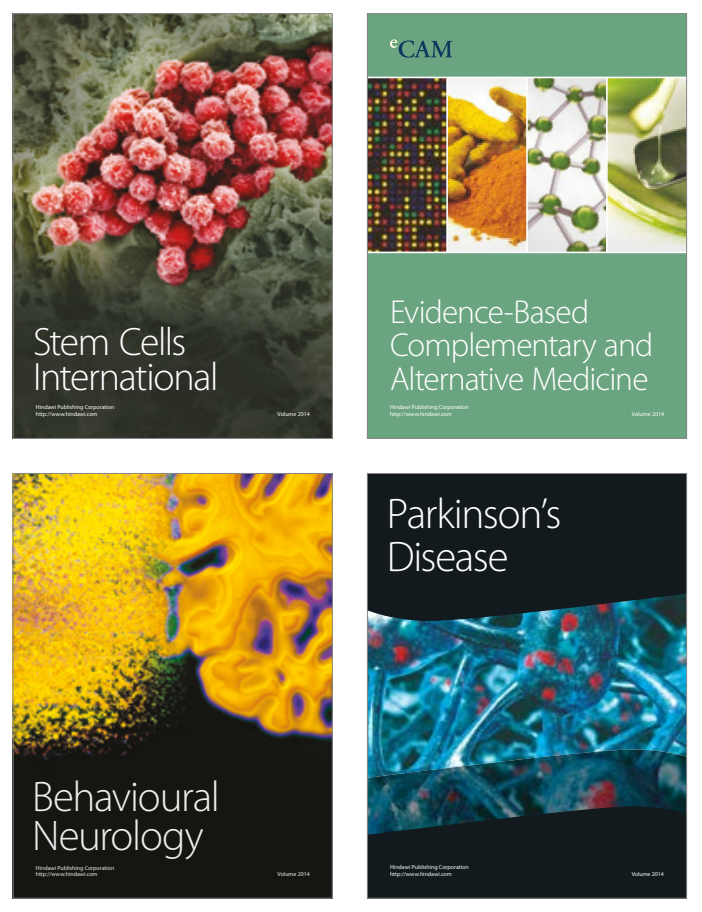
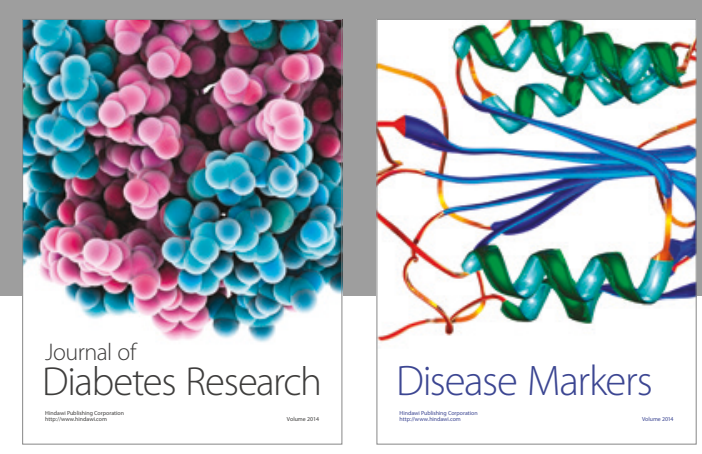

Disease Markers
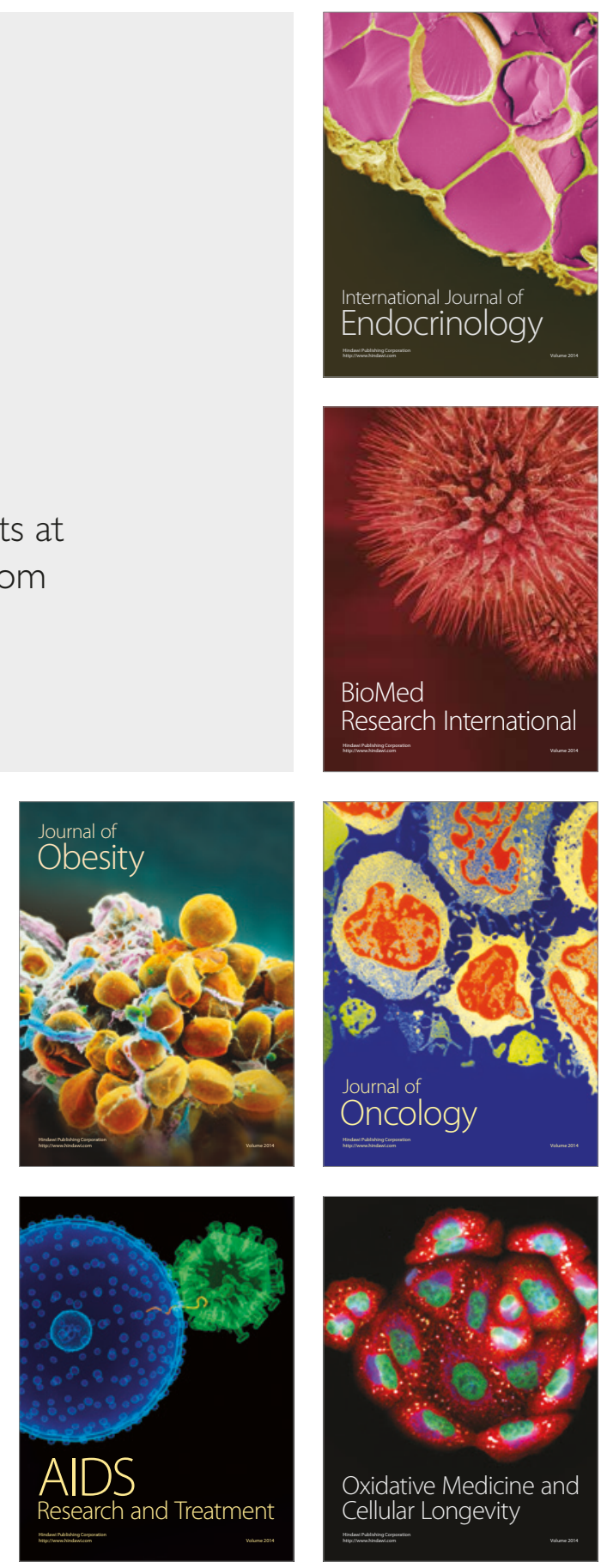